\title{
Performance Regulation of Event-Driven Dynamical Systems Using Infinitesimal Perturbation Analysis
}

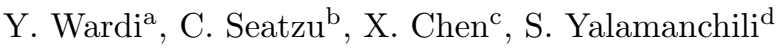 \\ ${ }^{a}$ School of Electrical and Computer Engineering, \\ Georgia Institute of Technology, Atlanta, Georgia, USA \\ (e-mail: ywardi@ece.gatech.edu). \\ ${ }^{b}$ Department of Electrical and Electronic Engineering, \\ University of Cagliari, Italy \\ (e-mail:seatzu@diee.unica.it). \\ ${ }^{c}$ School of Electrical and Computer Engineering, \\ Georgia Institute of Technology, Atlanta, Georgia, USA \\ (e-mail: xchen318@gatech.edu). \\ ${ }^{d}$ School of Electrical and Computer Engineering, \\ Georgia Institute of Technology, Atlanta, Georgia, USA \\ (e-mail:sudha@ece.gatech.edu).
}

\begin{abstract}
This paper presents a performance-regulation method for a class of stochastic timed event-driven systems aimed at output tracking of a given reference setpoint. The systems are either Discrete Event Dynamic Systems (DEDS) such as queueing networks or Petri nets, or Hybrid Systems (HS) with timedriven dynamics and event-driven dynamics, like fluid queues and hybrid Petri nets. The regulator, designed for simplicity and speed of computation, is comprised of a single integrator having a variable gain to ensure effective tracking under time-varying plants. The gain's computation is based on the Infinitesimal Perturbation Analysis (IPA) gradient of the plant function with respect to the control variable, and the resultant tracking can be quite robust with respect to modeling inaccuracies and gradient-estimation errors. The proposed technique is tested on examples taken from various application areas and modeled with different formalisms, including queueing models, Petri-net model of a production-inventory control system, and a stochastic DEDS model of a multicore chip control. Simulation results are presented in support of the proposed approach.
\end{abstract}

Keywords: Infinitesimal perturbation analysis, timed DEDS, stochastic hybrid systems, performance regulation.

${ }^{\sqrt{2}}$ Research supported in part by the NSF under Grant CNS-1239225. 


\section{Introduction}

This paper describes a regulation technique for a class of dynamical systems, designed for output tracking of a given setpoint reference. The regulator consists of an integral control with a variable gain, computed on-line so as to enhance the closed-loop system's stability margins and yield effective tracking. The gain-adjustment algorithm is based on the derivative of the plant's output with respect to its input control, and therefore the regulation technique is suitable for systems where such derivatives are readily computable in real time. This includes a class of stochastic timed Discrete Event Dynamic Systems (DEDS) and Hybrid Systems (HD) where the derivative is computable by the Infinitesimal Perturbation Analysis (IPA) sample-gradient technique. Our motivation is derived from the problem of regulating instructions' throughput in multicore computer processors, and following an initial study of that problem in Ref. [2] we extend the technique to a general class of DEDS and HS.

The need for regulating instruction throughput at the hardware level in modern computer processors stems from real-time applications where constant throughput facilitates effective real-time task and thread (subprogram) scheduling, as well as from multimedia applications where a fixed frame rate must be maintained to avoid choppy video or audio. The design of effective regulators is challenging because of the lack of predictive analytical or prescriptive models, and unpredictable high-rate fluctuations of instructions-related switching activity factors at the cores. For this reason, we believe, most of the published control techniques are ad hoc (see the survey in Ref. [15]). A systematic controltheoretic approach has been pursued in Refs. [4, 3, 15] which applied a PID controller and analyzed the effects of proportional controls with fixed gains. Concerned with the unpredictability and rapid changes in the thread-related activity factors, Ref. [2] sought a controller with adaptive gain. Furthermore, it considered scenarios where measurements and computations in the control loop must be performed as quickly as possible, even at the expense of accuracy. To this end it considered controlling the instruction throughput by a core's clock rate, and applied an integral controller whose real-time gain-adaptation algorithm is designed for stabilizing the closed-loop system and yielding effective tracking convergence. The gain-adaptation algorithm is based on IPA as described in the sequel.

An abstract, discrete-time configuration of the closed-loop system is shown in Figure 1, where $n$ denotes the time-counter, $r$ is the setpoint reference, $u_{n}$ is the control input to the plant, $y_{n}$ is the resulting output, and $e_{n}:=r-y_{n}$ is the error signal. The system is single-input-single-output so that all the quantities $u_{n}, y_{n}, e_{n}$ and $r$ are scalar.

Let $J: R \rightarrow R$ represent a performance function of the plant with respect to its input $u$, and assume that the function $J(u)$ is differentiable. Given the $n t h$ input variable $u_{n}$, suppose that the the plant's output $y_{n}$ provides an estimation of $J\left(u_{n}\right)$. The controller that we consider has the form

$$
u_{n}=u_{n-1}+A_{n} e_{n-1},
$$


and we recognize this as the discrete-time version of an integrator (summer) with a variable gain. As mentioned earlier, the gain sequence $\left\{A_{n}\right\}$ is designed to enhance the stability margins of the closed-loop system and reduce oscillations of the tracking algorithm while speeding up its convergence. As we shall see, one way to achieve that is to have $A_{n}$ be defined as

$$
A_{n}=\left(J^{\prime}\left(u_{n-1}\right)\right)^{-1}
$$

with "prime" denoting derivative with respect to $u$. However, it may not be possible to compute the derivative term $J^{\prime}\left(u_{n-1}\right)$, and approximations have to be used. Denoting the approximation error by $\phi_{n-1}$, the computed gain $A_{n}$ is defined as

$$
A_{n}=\left(J^{\prime}\left(u_{n-1}\right)+\phi_{n-1}\right)^{-1} .
$$

In the systems considered in this paper the plant represents average measurements taken from a physical system or a cyber system over contiguous timeintervals called control cycles. For example, suppose that the physical system is a continuous-time dynamical system with input $v(t)$ and output $\zeta(t), t \geq 0$; its state variable is immaterial for the purpose of this discussion. Divide the time axis into contiguous control cycles $C_{n}, n=1,2, \ldots$, suppose that the control input is fixed during $C_{n}$ to a value $u_{n}:=v(t) \forall t \in C_{n}$, and define $y_{n}$ by

$$
y_{n}:=\frac{1}{\left|C_{n}\right|} \int_{C_{n}} \zeta(t) d t,
$$

where $\left|C_{n}\right|$ is the duration of $C_{n}$. Alternatively, $y_{n}$ can represent average measurements taken from the output of a discrete-time or discrete-event system. Generally we impose no restriction on the way the control cycles are defined, they can be fixed a priori or determined by counting events in a DEDS; we only require that the input $u_{n}$ remains unchanged during $C_{n}$ and can be modified only when the next control cycle begins.

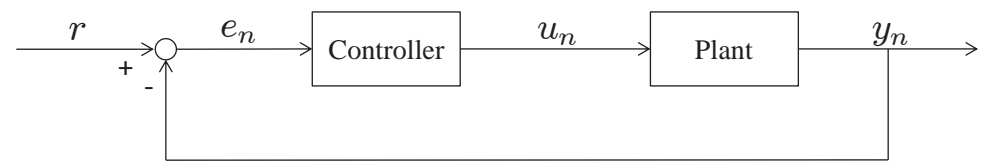

Figure 1: Basic regulation system

Observe that Eq. (3) suggests that the computation of $A_{n}$ takes place during the control cycle $C_{n-1}$. In fact, we assume that the implementation of the control law takes place in the following temporal framework. Suppose that the quantities $u_{n-1}$, and $y_{n-1}, e_{n-1}$, and $A_{n}$ have been computed or measured by the starting time of $C_{n}$. Then $u_{n}$ is computed from Eq. (1) at the start of $C_{n}$ and we assume that this computation is immediate. During $C_{n}$, the plant 
produces $y_{n}$ from the applied input $u_{n}$ while $A_{n+1}$ is computed from Eq. (3), with the index $n+1$ instead of $n$. Finally, $e_{n}$ is computed at the end of $C_{n}$ from the equation

$$
e_{n}=r-y_{n},
$$

and we assume that this computation is immediate.

The plant's actions yielding $y_{n}$ from $u_{n}$ during $C_{n}$ may represent a physical or cyber process or measurements thereof, and the computation of $A_{n+1}$ is assumed to be carried out concurrently. Of a particular interest to us is the case where $J\left(u_{n}\right)$ is an expected-value performance function of a DEDS or HS, $y_{n}$ is an approximation thereof computed from a sample path of the system, and the term $J^{\prime}\left(u_{n}\right)+\phi_{n}$ in the Right-Hand Side (RHS) of Eq. (3) (with $n+1$ ) is computable by IPA. One of the main appealing features of IPA is the simplicity of its gradient (derivative) algorithms and efficiency of their computation. This, however, comes at the expense of accuracy. In particular, in its principal application area of queueing systems during its earlier development, IPA often yielded statistically biased gradient estimators (see Refs. [11, 5]). To ameliorate this problem, Stochastic Flow Models (SFM) consisting of fluid queues (see Refs. $[6,22,7,17])$ and later extended to more general stochastic HS in Refs. $[8,24,25]$, offer an alternative framework to queueing networks for the application of IPA; in their setting the IPA gradients typically are simpler and more accurate. Still approximations must be made either in the IPA algorithms or in the system's model when a stochastic HS is used as a modeling abstraction for a DEDS. However, our overriding concern regarding the regulation's control law is that of simplicity and computational efficiency even if they come at the expense of accuracy. This is justified by sensitivity-analysis results, derived below, showing that asymptotic tracking of the regulation scheme holds under substantial relative errors in the gradient estimation.

The objective of this paper is to investigate the performance of our proposed tracking technique on a number of DEDS and HS by using the IPA method for computing the integrator's adaptive gain in the loop. In this we leverage on the simplicity and low computational efforts required for the IPA derivatives. Furthermore, simulation experiments suggest that the regulation algorithm works well despite substantial errors in the gradient estimation, thus allowing us to tilt the balance between precision and low computing times towards fast computation at the expense of accuracy. It may be asked why we use an integral control and not a PI or PID controller, and it is pointed out that we have tested via simulation (not reported here) the addition of a proportional element to the integral control, and found no improvement. This is not surprising since, as indicated by the analysis in Section 2, the particular gain-adaptation of the integral controller stabilizes the system for a class of plant functions $J(u)$. In summary, the contributions of this paper are: 1). It proposes the first generalpurpose, systematic performance-regulation technique for a class of timed DEDS and HS. 2). To-date, the main use of IPA has been in optimization, while this paper pursues a new kind of application, namely performance regulation. 3). It moves away from the traditional pursuit of unbiased IPA gradients, and instead 
searches for low-complexity approximations perhaps at the expense of accuracy or unbiasedness. We believe that these three points of novelty may open up a new dimension in the research and applications of IPA.

The rest of the paper is organized as follows. Section 2 describes the regulation technique in an abstract setting. The following three sections present simulation results on three types of systems, and highlight the tradeoffs between simplicity and accuracy. Section 3 concerns a queue, Section 4 considers a fluid Petri net production-control model, and Section 5 discusses a DEDS model of throughput in computer processors. Finally, Section 6 concludes the paper. ${ }^{1}$

\section{Regulation Algorithm in an Abstract Setting}

The rationale behind the choice of $A_{n}$ in Eq. (2) (if its RHS can be computed exactly) can be seen by considering the simple case where the plant consists of a deterministic, time-invariant, memoryless nonlinearity, and hence the $u_{n}$ - to $y_{n}$ relation has the form

$$
y_{n}=J\left(u_{n}\right) .
$$

Suppose that the function $J(u)$ is differentiable, and denote its derivative by $J^{\prime}(u)$. An application of the Newton-Raphson method for solving the equation

$$
r-J(u)=0
$$

results in the recursive equation

$$
u_{n}=u_{n-1}+\frac{1}{J^{\prime}\left(u_{n-1}\right)} e_{n-1}=u_{n-1}+A_{n} e_{n-1},
$$

where $A_{n}:=\left(J^{\prime}\left(u_{n-1}\right)\right)^{-1}$ is defined by (2). This yields Eq. (1), and we discern that the control law, comprised of repeated recursive applications of Eqs. $(2) \rightarrow(1) \rightarrow(5) \rightarrow(4)$, amounts to an implementation of the NewtonRaphson method.

An application of this control technique to a class of dynamic, time-varying systems is one of the main objectives of the present paper. Its convergence is underscored by established results on the sensitivity of the Newton-Raphson method with respect to variations in function and gradient-evaluations [14]. Therefore, the analysis of the paper next will be presented in an abstract setting of the Newton-Raphson method, and then related to the control configuration of Figure 1.

Let $g: R \rightarrow R$ be a continuously-differentiable function, and consider the problem of finding a root of the equation $g(u)=0, u \in R$. The basic step of the Newton-Raphson method is

$$
u_{n}=u_{n-1}-\frac{1}{g^{\prime}\left(u_{n-1}\right)} g\left(u_{n-1}\right),
$$

\footnotetext{
${ }^{1}$ The second queueing example in Section 3, and the Petri-net example in Section 4 were presented in, and are part of [19].
} 
and the algorithm is comprised of running this equation recursively for $n=$ $1,2, \ldots$ starting from an initial guess $u_{0} \in R$. Convergence of the algorithm can be characterized by the equation

$$
\lim _{n \rightarrow \infty} g\left(u_{n}\right)=0
$$

which implies that every accumulation point of the sequence $\left\{u_{n}\right\}, \hat{u}$, satisfies the equation $g(\hat{u})=0$.

Convergence of the Newton-Raphson method is well known under broad assumptions (see, e.g., Ref. [16]). Ref. [14] investigated the case where the derivative term $g^{\prime}\left(u_{n-1}\right)$ in Eq. (8) is approximated rather than evaluated exactly, and showed that convergence is maintained under suitable bounds on the errors. Taking it a step further, we consider the case where errors arise in the function evaluations $g\left(u_{n-1}\right)$ as well. Then Eq. (9) no longer can be expected, but (see [1]) the limit lim $\sup _{n \rightarrow \infty}\left|g\left(u_{n}\right)\right|$ is bounded from above by a term that depends on the magnitude of the errors in a suitable sense. Specifically, let $\psi_{n-1}$ and $\phi_{n-1}$ denote additive error terms in the computations of the function $g\left(u_{n-1}\right)$ and its derivative $g^{\prime}\left(u_{n-1}\right)$, respectively, so that Eq. (8) is transformed into

$$
u_{n}=u_{n-1}-\frac{1}{g^{\prime}\left(u_{n-1}\right)+\phi_{n-1}}\left(g\left(u_{n-1}\right)+\psi_{n-1}\right) \text {. }
$$

Define the relative errors $\mathcal{G}_{n-1}:=\frac{\left|\psi_{n-1}\right|}{\left|g\left(u_{-1}\right)\right|}$ and $\mathcal{E}_{n-1}:=\frac{\left|\phi_{n-1}\right|}{\left|g^{\prime}\left(u_{n-1}\right)\right|}$. The following results, Lemma 2.2 and Proposition 2.3, are proved under the following assumption. $^{2}$

Assumption 2.1. The function $g(\cdot)$ is continuously differentiable.

Given a closed interval $I:=\left[u_{1}, u_{2}\right] \subset R$, define $\left|g^{\prime}\right|_{I, \min }:=\min \left\{\left|g^{\prime}(u)\right|: u \in\right.$ $I\}$, and $\left|g^{\prime}\right|_{I, \max }:=\max \left\{\left|g^{\prime}(u)\right|: u \in I\right\}$. Consider the algorithm comprised of recursive runs of Eq. (10). For every $n=1, \ldots$, define

$$
m_{n}:=\min \left\{m>n: g\left(u_{m}\right) g\left(u_{n}\right) \geq 0\right\}
$$

in other words, if $g\left(u_{n}\right) \geq 0$ then $m_{n}$ is the next integer $m>n$ such that $g\left(u_{m}\right) \geq 0$, and if $g\left(u_{n}\right) \leq 0$ then $m_{n}$ is the next integer $m>n$ such that $g\left(u_{m}\right) \leq 0$.

Lemma 2.2. For every $M>1$ and $\beta \in\left(0, M^{-1}\right)$ there exist $\alpha \in(0,1)$ and $\theta \in(0,1)$ such that, for every closed interval $I$ where the function $g(u)$ has the following three properties: (i) $g(\cdot)$ is either monotone nondecreasing throughout I or monotone non-increasing throughout $I$; (ii) $g(\cdot)$ is either convex throughout I or concave throughout I; and (iii) $\frac{\left|g^{\prime}\right|_{I, \max }}{\left|g^{\prime}\right|_{I, \min }}<M$, the following holds: if,

\footnotetext{
${ }^{2} \mathrm{~A}$ weaker result than Lemma 2.2 and Proposition 2.3 was stated by Proposition 2 in [1], but its statement is incorrect.
} 
for some $n=1, \ldots$, (a) for every $j=n, \ldots, m_{n}, u_{j} \in I$; (b) for every $j=$ $n, \ldots, m_{n}-1, \mathcal{E}_{j}<\alpha$; and (c) for every $j=n, \ldots, m_{n}-1, \mathcal{G}_{j}<\beta$, then

$$
\left|g\left(u_{m_{n-1}}\right)\right|<\theta\left|g\left(u_{n-1}\right)\right| .
$$

The proof, tedious but based on standard arguments from convex analysis, is relegated to the appendix.

A few remarks are due.

1. In situations where $g(u)$ is the expected-value performance function of a DEDS or HS, it may be impossible to verify some of the assumptions underscoring Lemma 2.2, such as the continuous differentiability of $g(u)$, bounds on the relative errors $\mathcal{E}_{k-1}$ and $\mathcal{G}_{k-1}$, or bounds on the terms $g_{I, \max }^{\prime}$ and $g_{I, \text { min }}^{\prime}$. We point out that analysis techniques for their verifications have been developed in the literature on IPA, mainly for convex sample performance functions (see, e.g., [5, 7, 8]). However, in other situations these assumptions may have to be stipulated or justified by empirical evidence derived, for instance, from simulation.

2. Suppose that the variable $u$ has to be constrained to a closed interval $I:=$ $\left[u_{\min }, u_{\max }\right]$ satisfying the conditions of Lemma 2.2 . To ensure that $u_{n}$, $n=1, \ldots$ are contained in $I$, it is possible to modify Eq. (10) by following it with a projection onto $I$. Define the projection function $P_{I}: R \rightarrow I$ by

$$
P_{I}(u):= \begin{cases}u, & \text { if } u \in I \\ u_{\text {min }}, & \text { if } u<u_{\text {min }} \\ u_{\text {max }}, & \text { if } u>u_{\text {max }}\end{cases}
$$

and change Eq. (10) to the following equation,

$$
u_{n}=P_{I}\left(u_{n-1}-\frac{1}{g^{\prime}\left(u_{n-1}\right)+\phi_{n-1}}\left(g\left(u_{n-1}\right)+\psi_{n-1}\right)\right) .
$$

If $I$ contains a point $\hat{u}$ such that $g(\hat{u})=0$, in addition to satisfying the conditions of the lemma, then it is readily seen that using Eq. (12) instead of (10) does not weaken the statement of the lemma.

Proposition 2.3. For every $\eta>0, M>1$, and $\varepsilon>0$ there exist $\alpha \in(0,1)$ and $\delta>0$ such that, for every closed, finite-length interval I such that (i) throughout $I$ the function $g(\cdot)$ is either monotone increasing or monotone decreasing, and either convex or concave, (ii) the set $\{u \in I \mid g(u)=0\}$ is nonempty, and (iii) $\left|g^{\prime}\right|_{I, \text { min }}>\eta$ and $\frac{\left|g^{\prime}\right|_{I, \max }}{\left|g^{\prime}\right|_{I, \min }}<M$; and for every sequence $\left\{u_{n}\right\}_{n=1}^{\infty}$ computed by a recursive application of $E q$. (10) such that, for every $n=1,2, \ldots$, (a) $u_{n} \in I$, (b) $\mathcal{E}_{n}<\alpha$, and (c) $\left|\psi_{n}\right|<\delta$, the following two inequalities are in force:

$$
\limsup _{n \rightarrow \infty}\left|g\left(u_{n}\right)\right|<\varepsilon
$$

and

$$
\limsup _{n \rightarrow \infty}\left|g\left(u_{n}\right)+\psi_{n}\right|<\varepsilon
$$


Remarks:

1. Consider the case where $g(u)$ is computed exactly and the errors are only in its derivative. Then every interval $I$ and a sequence $\left\{u_{n}\right\}$ satisfying the conditions of the proposition also satisfy all the condition of Lemma 2.2, and hence, Eq. (11) for all $n=1, \ldots$ implies (9).

2. Notice that the conditions assumed in the proposition's statement include upper bounds on the absolute error of the function's estimation, $\left|\psi_{n-1}\right|$, and on the relative error in the derivative, $\mathcal{E}_{n-1}$. The reason for this discrepancy will be made clear in the proof of Proposition 2.3, where it will be shown that Eqs. (13) and (14) are due to such upper bounds.

3. In light of Remark 2 following Lemma 2.2, if an interval $I$ satisfying the conditions of Proposition 2.3 is a constraint set for the sequence $\left\{u_{n}\right\}$, then replacing Eq. 10) by (12) will not alter the assertions of the proposition expressed by Eqs. (13) and (14).

Consider the case where $g(u)$ is an expected-value performance function on a stochastic dynamical system, and suppose that Eq. (10) is run recursively in order to solve the equation $g(u)=0$. Suppose moreover that at the $n t h$ iteration the function $g\left(u_{n-1}\right)$ and its derivative $g^{\prime}\left(u_{n-1}\right)$ are estimated by sample averages over a control cycle $C_{n-1}$. Generally, even under conditions of stochastic stability and arbitrarily long control cycles, it is not true that $\{\mathcal{E}\}_{n-1}<\alpha$ and $\left|\psi_{n-1}\right|<\delta$ for all $n=1, \ldots$, as stipulated in the statement of Proposition 2.3. However practically, by the inequality in Eq. (11), with suitably-long control cycles we expect the sequence $\left\{\left|g\left(u_{n}\right)\right|\right\}$ to decline towards 0 as in (13) except for sporadic jumps away from zero, and this will be evident from the simulation results described in the next section. This is due to Lemma 2.2, while Proposition 2.3 provides a unified result under more ideal conditions.

In the context of the aforementioned control system, we note that the error in the derivative estimation is reflected by Eq. (3) rather than (2), and to account for the error in function evaluations, Eq. (5) is replaced by

$$
y_{n}=J\left(u_{n}\right)+\psi_{n}
$$

Now the control loop is defined by a recursive application of Eqs. $(3) \rightarrow(1) \rightarrow$ $(15) \rightarrow(4)$. To translate the algorithm defined by Eq. (1) into this control setting we define $g(u)=r-J(u)$. We can also apply it to time-varying systems of the form $y_{n}=J_{n}\left(u_{n}\right)$, where convergence in the sense of Eqs. (13) -(14) is expected if the systems vary slowly. Finally, we mention that from a practical standpoint the algorithm's convergence is ascertained from Eq. (14) rather than (13), since the observed quantity is $y_{n}=J\left(u_{n}\right)+\psi_{n}$ rather than $J\left(u_{n}\right)$.

The next section presents simulation examples of various DEDS and hybrid systems. They include two noteworthy situations concerning queueing systems where IPA is biased. The error term $\phi_{n-1}$ is due in one case to the use of SFM as a basis for the IPA formula, and in the other case, to a direct use of the DEDS-based IPA in spite of its bias. In both cases the regulation algorithm is 
shown to converge. While situations of the first case have stimulated an interest in SFM as a means to circumvent the bias inherent in IPA, the example of the second case demonstrates that IPA can be successfully used even when it is biased.

\section{Regulation of Average Delay and Loss Rate of a Single Queue}

This section illustrates the aforementioned regulation framework by applying it to two examples concerning, respectively, delay and loss rate in an M/D/1 queue. Both delay and loss are controlled by the service times. In the first example the IPA derivative is unbiased and hence we use it to adjust the integrator's gain. In contrast, in the second example the IPA derivative is biased and hence we apply a formula which is derived from an SFM (fluid-queue) approximation to the sample paths obtained from the discrete queue. While this yields estimation errors due to modeling discrepancies, it guarantees the unbiasedness of IPA for the SFM and results in convergence of the regulation algorithm as applied to the discrete queue.

\subsection{Average Delay}

Consider an $\mathrm{M} / \mathrm{D} / 1 / \infty$ queue with a given arrival rate $\lambda$ and service times of $s$ time-units. Given positive integers $M$ and $N$, a control cycle consists of $M$ jobs, and the regulation process is run for $N$ cycles. We set the queue to empty at the start of each control cycle. In the notation established in Section 1, we define $u=s$; for $n=1, \ldots, N, C_{n}$ is the $n t h$ control cycle; and $y_{n}$ is the average delay of jobs arriving during $C_{n}$. Note that $y_{n}$ is not an expected-value function but rather a random function of $u$ whose realization depends of the samples drawn during $C_{n}$. Let $J(u)$ denote the expected-value delay according to the stationary distribution. It is known that $y_{n}$ is a strongly-consistent estimator of $J\left(u_{n}\right)$ in the following sense (see $[11,5]$ ): for a given $n=1,2, \ldots$,

$$
\lim _{M \rightarrow \infty} y_{n}=J\left(u_{n}\right)
$$

where $M$ is the length of the control cycle. Consequently the estimation error $\psi_{n}:=J\left(u_{n}\right)-y_{n}$ can be made smaller by choosing longer control cycles.

The IPA derivative, $\frac{\partial y_{n}}{\partial u_{n}}$, is known to have the following form (see $[11,5]$ ). For every $m=1, \ldots, M$, let $k_{m, n}$ denote the index (counter) of the job that started the busy period containing job $m$ during $C_{n}$. Then,

$$
\frac{\partial y_{n}}{\partial u_{n}}=\frac{1}{M} \sum_{m=1}^{M}\left(m-k_{m, n}+1\right) .
$$

In other words, the IPA derivative of the delay of job $m$ as a function of $u_{n}$ is the position of that job in its busy period, namely $m-k_{m, n}+1$. This IPA is unbiased and strongly consistent; see Refs. [11, 5]. Furthermore, by the Pollaczek-Khinchin formula, $J(u)$ is a continuously-differentiable function of $u$. 
It is obviously monotone increasing, and also convex on $R^{+}$since, by Eq. (17), $J^{\prime}(u)$ is monotone increasing as well. Therefore the conditions for Proposition 2.3 are satisfied on any closed interval contained in $R^{+}$, and we expect the simulation experiments to yield fast convergence of the output $y_{n}$ to an $\varepsilon$-band around the target value $r$ (in the sense of Eq. (13)), where $\varepsilon$ can be made smaller by choosing longer control cycles.

In the first simulation experiment we set the arrival rate to $\lambda=0.9$, and the target reference delay to $r=3.0$. The control cycles consist of $M=10,000$ jobs each, and we ran the control algorithm for $N=100$ cycles starting from the initial guess of $u_{1}=1.1$. The results, shown in Figure 2, indicate an approach of $y_{n}$ to about a steady value in about 5 iterations. Thereafter we notice fluctuations of $y_{n}$, and a closer view of the results, obtained in Figure 3 by focusing the graph on the range $n=6, \ldots, 100$, shows them to be in the range of $2.5-3.5$ except for a single exception at $n=69$. These fluctuations do not seem to abate at larger $n$, and they likely are due to the variances of $y_{n}$ and its IPA derivative $\frac{\partial y_{n}}{\partial u_{n}}$. We point out that the average value of $y_{n}$, obtained over the range $n=6, \ldots, 100$, is 3.031 (recall that the target value is 3.0 ). Correspondingly, the graph of the service times $u_{n}, n=1, \ldots, 100$, is shown in Figure 4 , and their mean over $n=6, \ldots, 100$ is 0.913 .

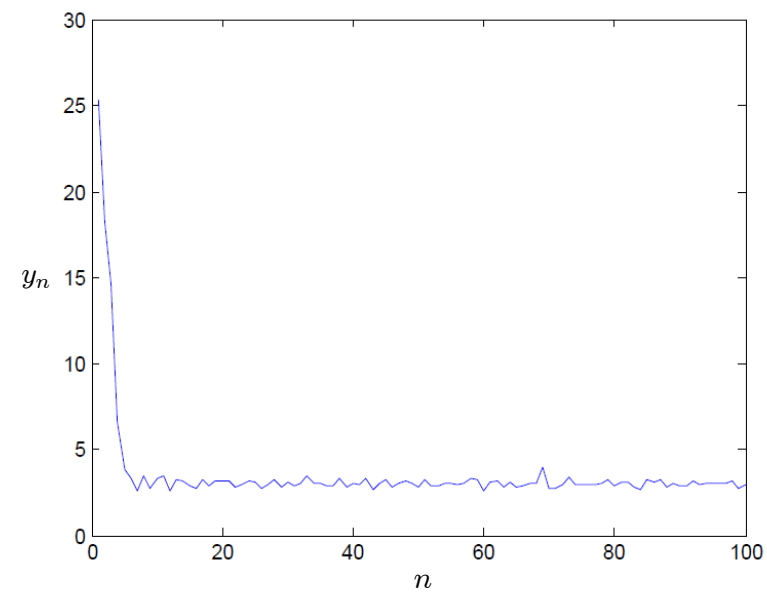

Figure 2: $M / D / 1 / \infty$ queue - delay. $M=10,000, n=1, \ldots, 100$.

Lesser variance of $\left\{y_{n}\right\}$ can be obtained from increasing the cycle length $M$ or reducing the reference target $y_{r e f}$. In the first case, where we set $M=100,000$ while keeping $y_{r e f}$ at $3.00, y_{n}, n=6, \ldots, 100$, fluctuated between 2.85 and 3.2 except for two values of $n$, and their mean was 3.008. In the second case, with $y_{\text {ref }}=1.5$ and $N=10,000, y_{n}$ fluctuated mostly in the [1.35-1.65]-range with 


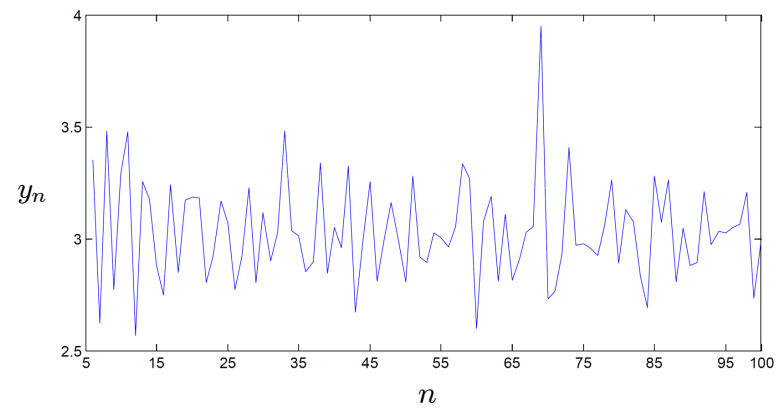

Figure 3: $M / D / 1 / \infty$ queue - delay, $n=6 \ldots, \infty . M=10,000, n=5, \ldots, 100$.

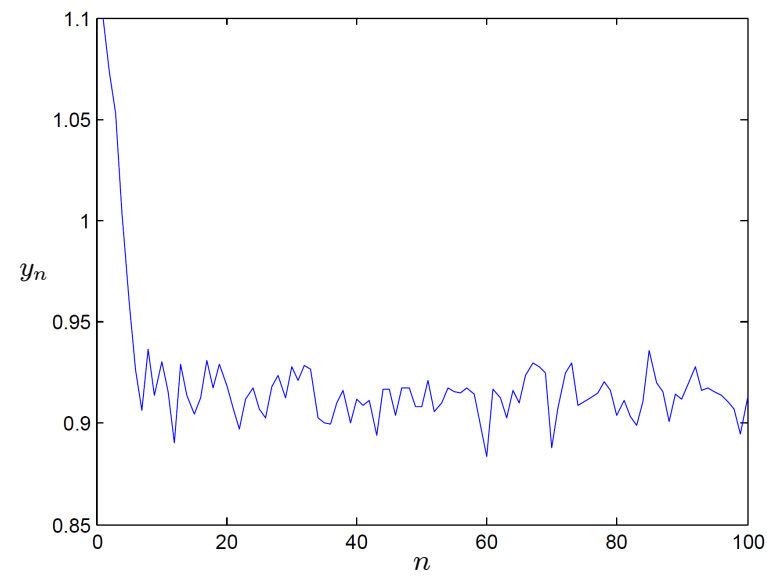

Figure 4: $M / D / 1 / \infty$ queue - service times. $M=10,000, n=1, \ldots, 100$.

two exceptions, and its mean over $n=6, \ldots, 100$ was 1.505 . In all three cases the regulation algorithm converged in about 5 iterations independently of the variances of the delays and their IPA derivatives; those seem to affect mostly the magnitude of the output fluctuations.

In order to test the effects of changing the target reference during an experiment, we ran the regulation algorithm for 120 cycles starting with $u_{1}=1.1$; in the first 40 cycles $y_{r e f}=3.0$, in the next 40 cycles $y_{r e f}=4.5$, and in the last 40 cycles, $y_{r e f}=1.5$. The results are shown in Figure 5, and they indicate convergence to each value of $y_{r e f}$ in about 5 iterations. Furthermore, it is evident that the variations are larger for larger $y_{r e f}$, and the reason is that the service times converge to larger values and hence the queue has larger traffic intensities. For $y_{r e f}=3.0$ the mean of $y_{n}$ over $n=5, \ldots, 40$ is 3.052 , for $y_{r e f}=4.5$ the mean of $y_{n}$ over $n=45, \ldots, 80$ is 4.653 , and for $y_{\text {ref }}=1.5$ the mean of $y_{n}$ over $n=85, \ldots, 120$ is 1.504 . The corresponding average gains $A_{n}$ obtained from the simulation were $0.063,0.030$, and 0.198 . The respective average service 
times were $0.918,0.977$, and 0.745 . These values correspond to traffic intensities (product of $\lambda$ and the service time) of $0.826,0.879$, and 0.671 , which explain the noticeable differences in variability. When we took $M=100,000$ the variability declined (as expected) and the obtained means are 3.041, 4.515, and 1.501, respectively.

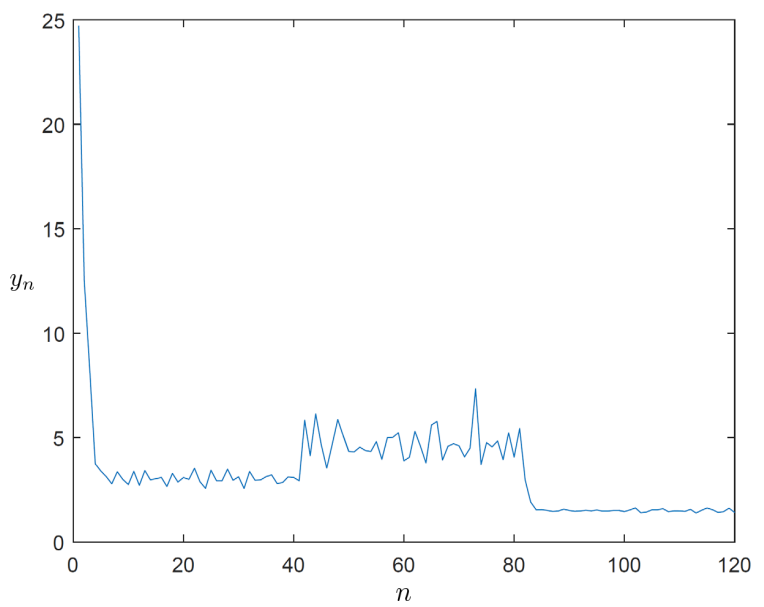

Figure 5: $M / D / 1 / \infty$ queue - delay, variable setpoint. $M=10,000, n=1, \ldots, 120$.

Lastly, we demonstrate the advantage of using the variable-gain controller over fixed-gain integral controls. To this end we simulated the system described in the last paragraph with two fixed gains, and compared the results to those obtained earlier from the variable-gain control. The constant gains are 0.030 and 0.198 which, as reported above, are the average gains driven by the variable-gain controller for the respective extreme setpoint references of 4.5 and 1.5.

Figure 6 depicts the graph of $y_{n}, n=5, \ldots, 120$, obtained from an application of the smaller fixed gain of 0.030 (the dashed curve), while the analogous graph obtained from the variable-gain controller (the solid curve) is shown for the sake of comparison. We discern slower response of the fixed-gain controller to changes in the setpoint, and this is not surprising since the variable-gain controller has larger gains for the setpoints of 3.0 and 1.5. For the larger fixed gain of 0.198 , the graph of $y_{n}$ is shown in Figure $7 .^{3}$ The controller performs well for the setpoint of 1.5, but it exhibits large oscillations suggesting instability for the setpoint of 4.5, where the obtained gains of the variable-gain controller are smaller. These results are not surprising since, generally, tracking controllers with too-small gains may result in slow adjustment to variations in the target

\footnotetext{
${ }^{3}$ This graph could not be adequately shown in the same figure as the graphs in Figure 6 due to the large peaks of $y_{n}$.
} 
setpoint, while overly-large gains may result in oscillations and even instability. The variable-gain controller in this example seems to adjust well to changes in the setpoint and thus perform better than the considered fixed-gain controls.

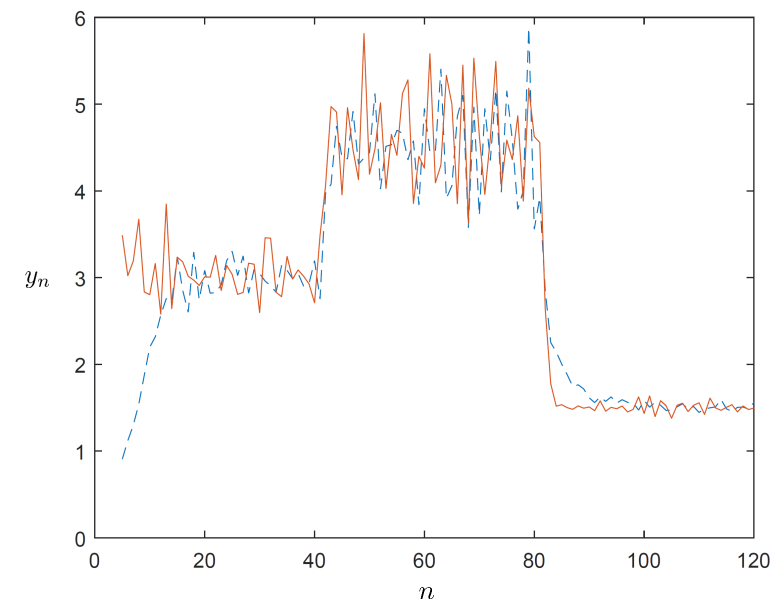

Figure 6: Comparison of a small, fixed-gain control to the variable-gain controller.

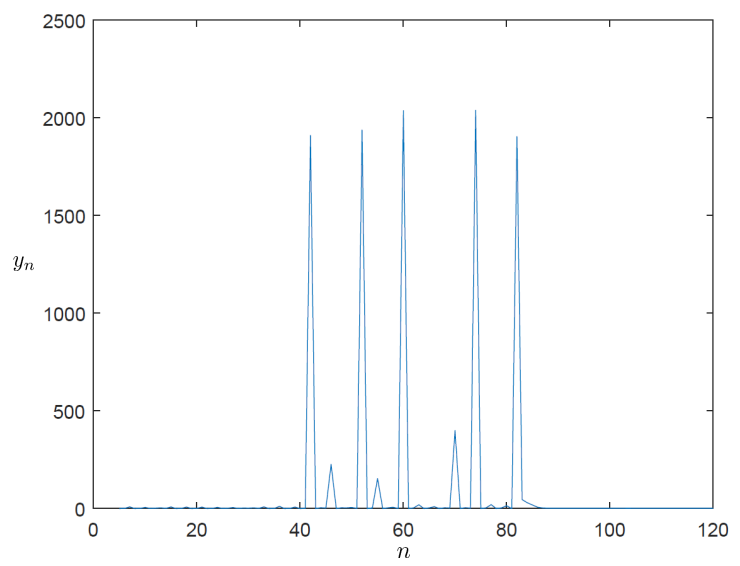

Figure 7: Oscillations due to a large, fixed-gain control.

\subsection{Average loss-rate}

Consider an $\mathrm{M} / \mathrm{D} / 1 / \mathrm{k}$ queue with a finite buffer, where jobs arriving at a full queue are being discarded. Given an arrival rate, a buffer size, and a horizon 
period (cycle time), we aim at controlling (regulating) the mean loss rate to a given reference by adjusting the service times. Accordingly, we denote the arrival rate by $\lambda$, the service times by $s$, the buffer size (including the holding place at the server) by $k$, and the horizon period by $t_{f}$.

Let us divide the time-axis into consecutive control cycles, $C_{n}$, each of duration $t_{f}$ seconds. The control parameter is the service time, namely $u=s$, and during $C_{n}$ its value is denoted by $u_{n}$. The performance function of interest, $J(u)$, is the mean loss rate per control cycle. We approximate $J\left(u_{n}\right)$ by $y_{n}$, defined as the number of jobs discarded during $C_{n}$ divided by $t_{f}$.

It is readily seen that the function $u_{n} \rightarrow y_{n}$ is piecewise constant and almost surely no arrival would occur at the same time when another job enters or exits the server, and therefore, the IPA derivative along a sample path is $\frac{d y_{n}}{d u_{n}}=0$. This does not provide an adequate approximation to $J^{\prime}\left(u_{n}\right)$, and hence an alternative approach to the estimation of $J^{\prime}\left(u_{n}\right)$ is needed. To this end we use a fluid-queue SFM as described in the next paragraph.

Consider a fluid queue with a finite buffer, a time-varying inflow rate, and a constant service rate. Suppose that the queue operates in a given time-interval $\left[0, t_{f}\right]$, where its instantaneous arrival rate, denoted by $\alpha(t)$, is a random process. Denote its service rate by $\beta$. Let $u:=\beta^{-1}$ be the control variable, and let $\gamma(u, t)$ denote the instantaneous overflow (spillover) rate due to the limited buffer. Let $L(u)$ denote the sample-based average loss rate per cycle as a function of the input service rate $u$, defined as

$$
L(u)=\frac{1}{t_{f}} \int_{0}^{t_{f}} \gamma(u, t) d t .
$$

Observe that $L(u)$ provides an approximation to $J(u)$ to the extent that the fluid queue serves to approximate the discrete queue. The main difference between $J(u)$ and $L(u)$ is that $J(u)$ is the mean loss rate of the discrete queue, while $L(u)$ is the sample time-average loss rate per cycle of the continuous queue.

Reference [6] showed that the IPA derivative $L^{\prime}(u)$ is unbiased, and it is computable by a simple, model-free formula (listed below) that can act on the sample paths of the discrete queue. Furthermore, it can serve as an approximation to $J^{\prime}(u)$. Therefore we implement the regulation algorithm in the following way. During cycle $C_{n-1}$, the service time is $u_{n-1}, y_{n-1}$ is the resulting sample-average loss rate of the discrete queue, and $\psi_{n-1}:=y_{n-1}-J\left(u_{n-1}\right)$. The IPA derivative $L^{\prime}\left(u_{n-1}\right)$, specified below, is the sample derivative of the average loss rate of the continuous queue as defined by Eq. (18), and $\phi_{n-1}:=L^{\prime}\left(u_{n-1}\right)-J^{\prime}\left(u_{n-1}\right)$. Eq. (3) becomes

$$
A_{n}:=\left(L^{\prime}\left(u_{n-1}\right)\right)^{-1} \text {. }
$$

In this we apply the IPA derivative-formula, obtained from an analysis of the $\mathrm{SFM}$, to the sample path of the discrete queue during $C_{n-1}$. In contrast, the plant's output $y_{n-1}$ that is used in the control loop via Eq. (1) with $e_{n-1}:=$ $r-y_{n-1}$ corresponds to the discrete queue since it represents the "real" system. The effectiveness of the resulting regulation algorithm is related to the quality of the approximation of $J\left(u_{n}\right)$ and $J^{\prime}\left(u_{n}\right)$ by $y_{n}$ and $L^{\prime}\left(u_{n}\right)$, respectively. Now 
Ref. [6] showed that the function $L(u)$ is Lipschitz continuous in $u$, w.p.1. Furthermore, by Eq. (20), below, $L^{\prime}(u) \geq 0$, and $L(u)$ and $L^{\prime}(u)$ are monotone increasing and hence the function $L(u)$ is convex w.p.1. Therefore we believe that $J(u)$ is convex as well although we do not know of a proof, and in this case the assumptions of Lemma 2.2 and Proposition 2.3 are in force. In any event, the effectiveness of the regulation algorithm will be tested by simulation.

The IPA derivative $L^{\prime}(u)$ has the following form (see [6]). Suppose that there are $Q$ lossy busy periods during the horizon interval $\left[0, t_{f}\right]$, indexed by $q=1, \ldots, Q$ in increasing order (a busy period is lossy if any positive amount of overflow is incurred throughout its duration). For the qth lossy busy period, let $u_{q}$ be the first time loss occurs during it, and let $v_{q}$ be its end point; in other words, $u_{q}$ is the first time in that busy period when the buffer becomes full, and $v_{q}$ is the next time the buffer becomes empty. Then, under mild assumptions $[6]$,

$$
L^{\prime}(u)=\frac{1}{t_{f}} u^{2} \sum_{q=1}^{Q}\left(v_{q}-u_{q}\right) .
$$

With $A_{n}$ defined by (19), we ran a simulation with the following parameters: $y_{\text {ref }}=0.1, t_{f}=4,000, k=3, \lambda=0.9$, the initial parameter-value was $u_{1}=1.5$, and the number of cycles was $N=100$. The resulting graph of $y_{n}, n=1, \ldots, 100$ is shown in Figure 8, where we notice convergence of the tracking algorithm in 3 iterations, to a band around the target value of 0.1 . Within this band $y_{n}$ fluctuates between 0.08 and 0.125 , except for a single value of $n$ where $y_{n}=$ 0.139 . The mean of $y_{n}$ in the range $n=5, \ldots, 100$ was 0.1005 . To reduce the variability we ran the simulation for $t_{f}=20,000$, and the results, shown in Figure 9, exhibit an equally-fast convergence of the regulation algorithm with fluctuations in the range of $[0.091,0.114]$, and with mean (over $n=5, \ldots, 100$ ) of 0.1000 .

\section{Application to a production system modeled by a Petri net}

The IPA technique recently has been extended from fluid queueing networks to a class of continuous Petri nets $[28,26]$. References $[26,18]$ applied the results to an optimization example of balancing part-inventories with product backorders in a single-stage manufacturing system, and tested the application of IPA in conjunction with a stochastic approximation algorithm. This section uses the same example to test our approach to regulation.

The considered manufacturing system consists of a machine that produces a sequence of single-type products. The production schedule is driven by products' orders while parts' inventories are maintained as safety stocks. To make a product, the system must have an available part and a product order; parts without orders accumulate in the form of inventories, while orders without parts 


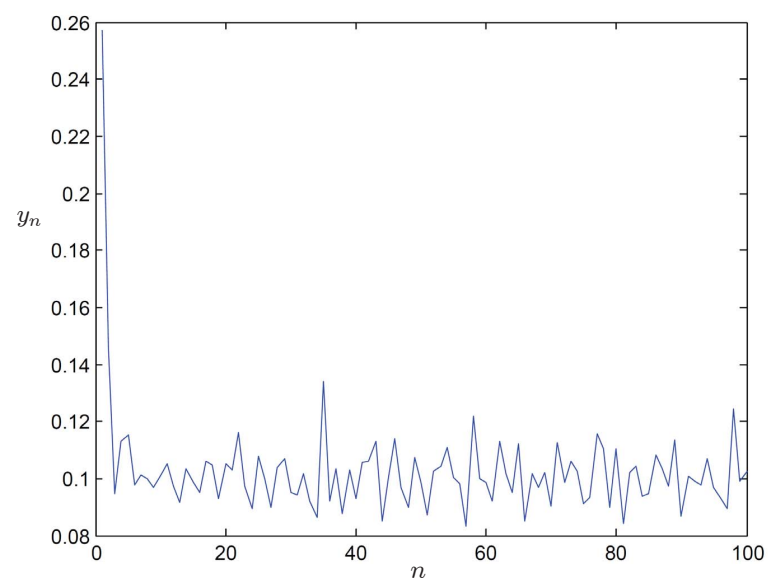

Figure 8: M/D/1/k queue - loss. $t_{f}=4,000, n=1, \ldots, 100$.

result in cumulative backorders. Naturally both excessive inventories and backorders are undesirable, and References [26, 18] devise an IPA-based algorithm for optimally balancing them. The underlying model for the algorithm is comprised of the continuous (fluid) Petri net (event graph) shown in Figure 10.

Continuous Petri nets are hybrid Petri nets where the flow of fluid tokens through transitions is represented by piecewise-continuous rate processes; see, e.g., [21] for comprehensive presentations. With regard to our system shown in Figure 10, transitions $T_{1}, T_{2}$, and $T_{3}$ represent, respectively, the processes of product-orders, parts' arrivals, and the machine's operation. Each transition $T_{i}$, $i=1,2,3$, is characterized by a maximum fluid-flow rate $V_{i}(t)>0$, which acts as an upper bound on its actual flow rate, denoted by $v_{i}(t)$. The places $p_{1}$ and $p_{2}$ are used for holding fluid, and at time $t$ the amount of stored fluid is denoted by $m_{1}(t)$ and $m_{2}(t)$, respectively. The processes $\left\{V_{i}(t)\right\},\left\{v_{i}(t)\right\}(i=1,2,3)$ and $\left\{m_{j}(t)\right\}(j=1,2)$ can be viewed as random processes defined over a common probability space $(\Omega, \mathcal{F}, P)$.

The dynamics of the system are described by the following three equations relating the above processes: For $i=1,2$,

$$
v_{i}(t)=V_{i}(t)
$$

For $i=3$, define $\varepsilon_{3}(t):=\left\{j \in\{1,2\}: m_{j}(t)=0\right\}$; then

$$
v_{3}(t)= \begin{cases}V_{3}(t), & \text { if } \varepsilon_{3}(t)=\emptyset \\ \min \left(v_{i}(t): i \in \varepsilon_{3}(t)\right), & \text { if } \varepsilon_{3}(t) \neq \emptyset\end{cases}
$$

As for $m_{j}(t), j=1,2$, we have that

$$
\dot{m}_{j}(t)=v_{j}(t)-v_{3}(t)
$$




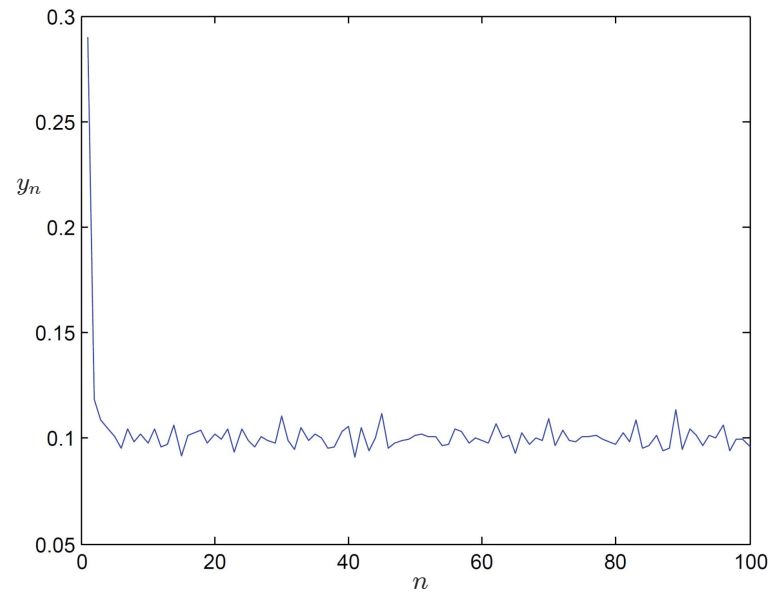

Figure 9: M/D/1/k queue - loss. $t_{f}=20,000, n=1, \ldots, 100$.

In the forthcoming discussion we assume that the system evolves in a given time-interval $\left[0, t_{f}\right]$ with given initial conditions $m_{1}(0)$ and $m_{2}(0)$.

In the typical case where the three processes $\left\{V_{i}(t)\right\}$ are exogenous, the other network processes, $\left\{v_{i}(t)\right\}$ and $\left\{m_{j}(t)\right\}$, are defined in their terms via Eqs. (21)(23). In other situations some of the processes $\left\{V_{i}(t)\right\}$ are exogenous while others are controlled, and in this case the equations describing the controls together with (21)-(23) define all of the network processes. In the example considered in $[26,18]$ the processes $\left\{V_{1}(t)\right\}$ and $\left\{V_{3}(t)\right\}$ are exogenous while $\left\{V_{2}(t)\right\}$ is controlled. Specifically, product orders are assumed to arrive in batches, and hence

$$
V_{1}(t)=\sum_{k \geq 1} \alpha_{k} \delta\left(t-s_{k}\right)
$$

where $\delta(\cdot)$ is the Dirac delta function, $s_{k}, k=1,2, \ldots$, are the arrival times, and $\alpha_{k}$ represent the quantities of the orders. The machine is assumed to have deterministic service times, and hence $V_{3}(t)=V_{3}$ for a given $V_{3}>0$. The parts' arrival rates are controlled by the backorders via a threshold in the following fashion: $V_{2}(t)$ has a given low value if the backorder levels are below the threshold, and a given higher value if the backorder levels are above the threshold. Formally, given a threshold $\rho>0$, and given constants $V_{2,1} \geq 0$ and $V_{2,2}>V_{2,1}, V_{2}(t)$ is defined via

$$
V_{2}(t)= \begin{cases}V_{2,1}, & \text { if } m_{1}(t) \leq \rho \\ V_{2,2}, & \text { if } m_{1}(t)>\rho .\end{cases}
$$

We assumed that $V_{2,1} \leq V_{3} \leq V_{2,2}$. It is obvious that Equations (21)-(23) and (25) have a unique joint solution for every set of initial conditions.

Now let us consider the threshold $\rho$ as the control parameter and hence denote it by $u=\rho$. Then the processes $\left\{V_{2}(t)\right\},\left\{v_{i}(t)\right\}, i=2,3$, and $\left\{m_{j}(t)\right\}$, 


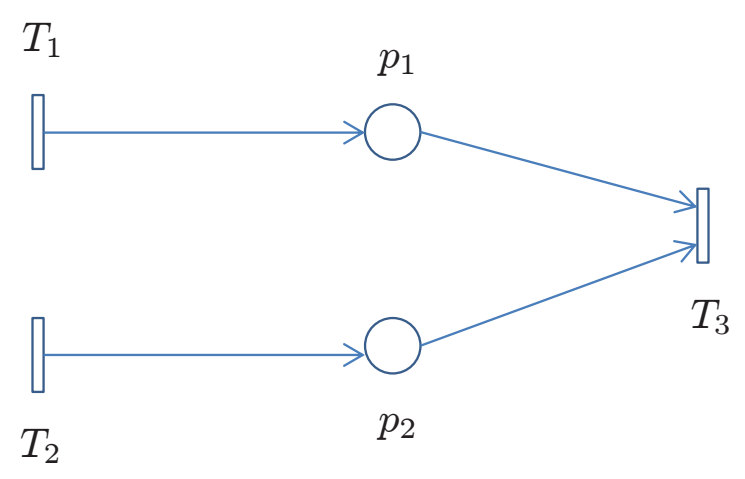

Figure 10: Basic event graph

$j=1,2$ are functions of $u$ as well, and hence are denoted by $\left\{V_{2}(u, t)\right\},\left\{v_{i}(u, t)\right\}$, and $\left\{m_{j}(u, t)\right\}$, respectively. Assume that a particular value of $u$ remains fixed throughout the evolution of the system in a given interval $\left[0, t_{f}\right]$. Consider the sample performance function $L(u)$ defined as

$$
L(u)=\frac{1}{t_{f}} \int_{0}^{t_{f}} m_{2}(u, t) d t,
$$

for a given distribution of the initial conditions $m_{j}(0), j=1,2$, and let $J(u):=$ $E(L(u))$ denote its expected value. References [26, 18] considered the problem of minimizing a weighted sum of $J(u)$ and the expected-value of the average workload at $p_{1}$. Here we use the same system except that we perform regulation of $L(u)$ rather than optimization.

To put it all in the setting described in Section 1, we define a control cycle to consist of $t_{f}$ time units, $u_{n}$ denotes the input during the $n t h$ control cycle $C_{n}$, and $y_{n}:=L\left(u_{n}\right)$ as defined by Eq. (26). Therefore, for every $n=1, \ldots$, $y_{n} \rightarrow J\left(u_{n}\right)$ as $t_{f} \rightarrow \infty$ w.p.1. Moreover, Refs. [26, 18] prove that the IPA derivative $L^{\prime}(u)$ is unbiased, hence $\frac{\partial y_{n}}{\partial u_{n}} \rightarrow_{t_{f} \rightarrow \infty} J^{\prime}\left(u_{n}\right)$ w.p.1. Consequently, the error terms $\psi_{n}$ and $\phi_{n}$ can be reduced by taking longer cycle times $t_{f}$. Regarding monotonicity and convexity of $J(u)$, larger threshold $u$ results in smaller inventories and hence $J(u)$ is monotone non-increasing. However, we have no way of proving convexity or concavity of $J(u)$, hence we put the regulation algorithm to the test by simulation.

In the considered example, $V_{3}=6, V_{2,1}=2.15$, and $V_{2,2}=6$; these numbers are taken from $[26,18]$. The product-orders process $\left\{V_{1}(t)\right\}$, defined by (24), consists of equally-spaced arrivals every 50 seconds (deterministic), and each arrival brings in an amount of fluid that is uniformly distributed in the $[30,70]$-range. The reference value to be tracked is $J_{\text {ref }}=758.70$, and it is the computed value of $J$ obtained for the aforementioned optimization problem in [18]. The IPA derivative $L^{\prime}(\theta)$ is computable via a recursive algorithm con- 
structed according to the event-calculus framework defined in $[8,26]$; a detailed presentation thereof can be found in [18].

We ran the regulation algorithm for 100 control cycles with $t_{f}=1,000$. The results of two typical runs are shown in Figure 11 for two respective values of the initial control parameter, $u_{1}=35$ and $u_{1}=15$. The corresponding graphs of $y_{n}:=L_{n}\left(u_{n}\right)$ are plotted by the dashed curve and solid curve, and both indicate convergence to a band around $y_{\text {ref }}=758.70$ after 3 iterations. This band has a maximum range of 47.17 , and the averages of the outputs $y_{n}$, taken over $n=20, \ldots, 100$, are 758.55 for the dashed plot, and 758.73 for the solid plot. Although these results indicate convergence of the outputs' average to $y_{\text {ref }}$, variations in the output values are discernable. These variations, as well as those in the IPA derivatives, yield fluctuations in the values of $u_{n}, n=1,2, \ldots$, as can be seen in Figure 12. We believe that the major cause of these variations is in the variance of $L(u)$ and not inherent in the regulation algorithm. To test this point, we ran 100 independent simulations of the system at the fixed value of $u=24.8$, which is close to the average of $u_{n}, n=20, \ldots, 100$ obtained by the regulation algorithm. The results, $\{L(u)\}_{i}$, shown in Figure 13, indicate a persistent variation with a maximum range of 42 , which is comparable to the range obtained from Figure 11.

To further test the convergence rate of the regulation algorithm we chose more extreme starting values of the control parameter, namely $u_{1}=45$ and $u_{1}=5$. The corresponding values of $y_{1}$ are 635 and 883 , which are more extreme than those obtained in Figure 11, but nonetheless the regulation algorithm converged to a similar band around $y_{\text {ref }}$ in 3 iterations.

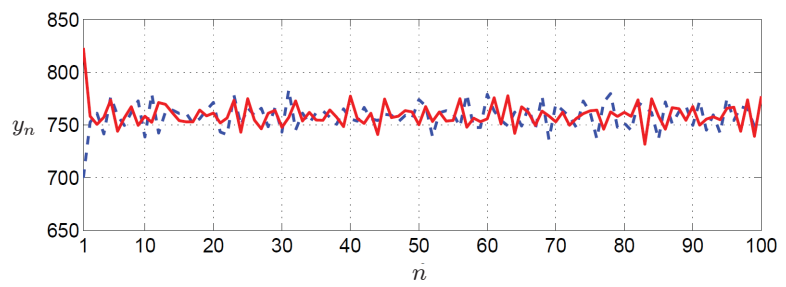

Figure 11: Petri net - inventory. $t_{f}=1,000, n=1, \ldots, 100$

\section{Application to throughput regulation in computer processors}

The problem of maintaining stable instruction execution rates in computer processors arises in several application areas. For instance, in real-time applications, guaranteeing a constant instruction execution rate simplifies otherwise complex real-time scheduling tasks by enabling tighter bounds on deadlines to be met [20]. Variable rate instruction execution often leads to a reliance on worst 


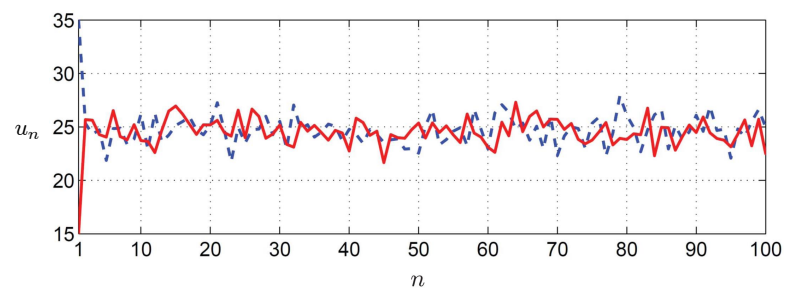

Figure 12: Petri net - threshold control. $t_{f}=1,000, n=1, \ldots, 100$

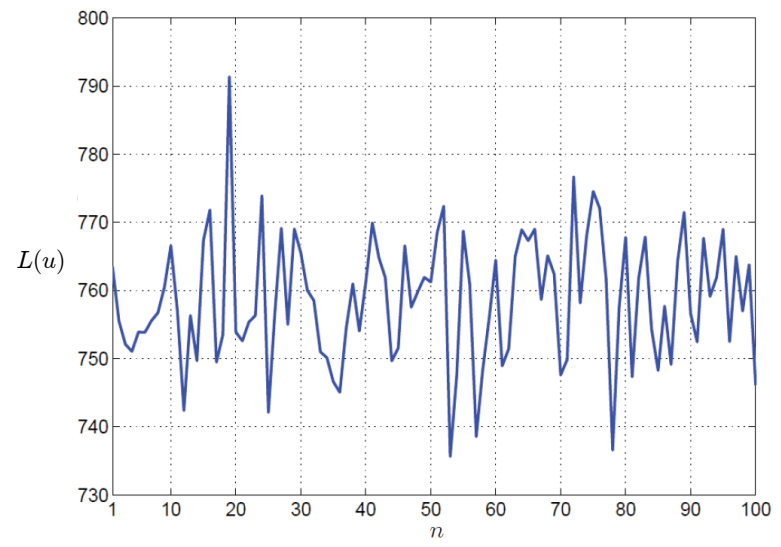

Figure 13: Variations in $L(u): 100$ independent simulations

case execution-time estimates that far exceed the average execution times, resulting in a poorer processor utilization than is necessary. A second application concerns a tradeoff between instruction execution rate and power dissipation. Dynamic Voltage Frequency Scaling (DVFS) can be used to navigate this tradeoff to arrive at an instruction rate that is the most power-efficient [12][13]. This approach, described in the Introduction, was followed by [2] for throughput regulation in processor cores. In this section we pursue a similar approach but use a more-precise system-model for the throughput simulation and hence obtain better results, including far-faster convergence, as described in the sequel.

We consider a multiprogrammed multi-core processor where programs are assigned for execution to the cores by the operating system. Each core is assigned an instruction-execution rate setpoint by a supervisory controller, and has to control (regulate) its instruction rate to that level. In this paper we are not concerned with the way these setpoint levels are assigned, and consider them as given and fixed. Furthermore, we assume that each core is in a separate clock domain and can independently control its own clock rate. Each core exploits instruction-level parallelism (ILP) utilizing an Out-of-Order (OOO) technology whereby instructions may complete execution in an order different from program order (i.e., out of order). OOO execution enables instruction execution 
to be limited only by data dependencies rather than the order in which they appear in the program thereby serving as the primary means for exploiting ILP in modern processors.

A high-level functional and logical description of programs' execution in an OOO core is depicted in Figure 14. Instructions are fetched sequentially from memory and placed in the Instruction queue. There they are processed by functional units which can be thought of as servers in the queueing parlance. It is often the case that there are enough functional units available for concurrent execution of all of the instructions in the queue. What holds up the execution of an instruction is its dependency on data that would become available from the execution of another instruction. For example, the instruction of adding two variables, $x$ and $y$, requires that both variables have numerical values. $x$ may be obtained from another numerical instruction, while $y$ may be fetched from memory. Thus, it is evident that the addition of $x$ and $y$ cannot commence before the instructions of computing $x$ and fetching $y$ are completed.

Instructions can be classified as data dependent vs. data independent according to whether they depend on data provided by the execution of other instructions. If an instruction is data dependent then its execution can commence as soon as all such data and a functional unit become available. If the instruction is data independent then its execution can start as soon as a functional unit is available.

Memory instructions can take one-to-two orders of magnitude more time to execute than computational instructions. Therefore most architectures make use of a hierarchical memory arrangement where on-chip cache access takes less time than external memory such as DRAM. First the cache is searched, and if the variable is found there then it is fetched and the instruction is completed. If the variable is not stored in cache then a cache miss occurs and a cache line (containing the variable) is fetched from external memory (typically DRAM) and placed in the cache. The variable is then accessed and the instruction is completed. We can think of all external-memory (non-cache) accesses as placed in a First-in-First-out queue designated by the Memory box in the figure. Furthermore, this queue has a finite buffer, and when it becomes full the entire memory access, including from the cache, is stalled.

During its processing the instruction is still stored in the Instruction queue, where it has been placed since its arrival at the start of the aforementioned process. Although it may be executed concurrently with other instructions subject to data-dependency constraints, it may not be released from the queue upon completion of its execution; in fact, its release time is the later of its completion time and the release time of the previous instruction. However, the variables it computes become available to other instructions upon its completion and not release. We point out that the term release used here is called commitment in the parlance of computer architectures. It is this quantity that is used to compute the instruction's throughput, as will be made clear in the sequel.

A high-level description of the hardware of an OOO core is depicted in Figure 15 (see [10] for a more detailed description). The acronyms in the various blocks indicate the following entities: IQ - Instruction Queue; ROB - Reorder 


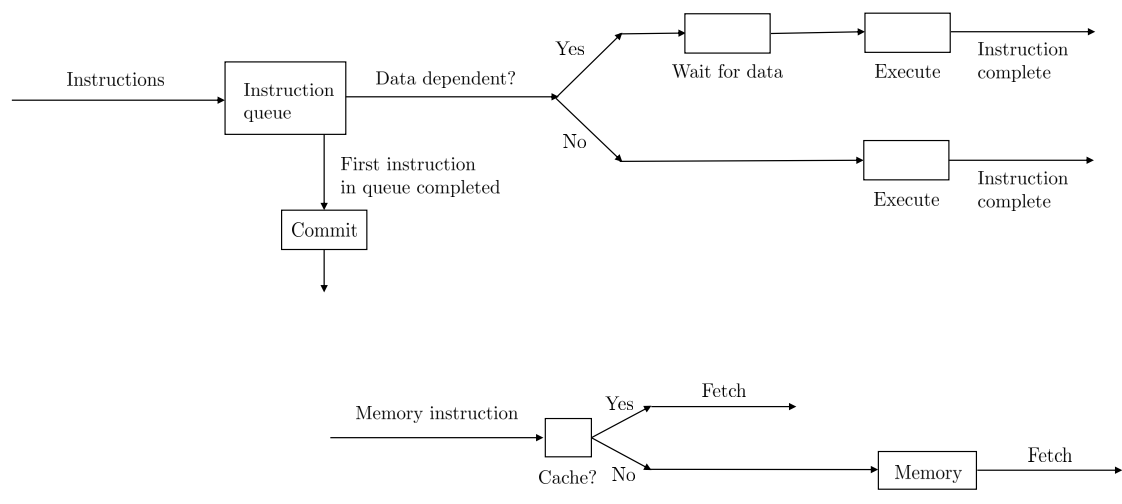

Figure 14: Functional description of OOO processing

Buffer; RS - Reservation Station; FU - Functional Unit; RF - Register File; L/S Q - Load/Store Queue; MSHR - Miss Status Handling Register; and MEM - memory other than cache, typically DRAM. During a program's execution, the instruction unit of the processor fetches program instructions sequentially from memory and places them in the Instruction Queue (IQ). Instructions are issued from the IQ to a reservation station waiting for access to the corresponding functional unit. An instruction is granted access (starts processing) after (i) all its operands are available, for example, the result from preceding instructions, and (ii) the functional unit is available. When an instruction is issued to an RS, it is also allocated an entry in the ROB in the original program order.

Instructions in the RS are issued to the functional units when their operands are available (note that this issue order may be different from program order hence the OOO designation). While the instruction is waiting for its operands, it is said to be stalled. Instruction execution results are broadcast to all reservation stations and the ROB - they are stored along with the instruction's entry in the ROB. When an instruction reaches the head of the ROB and it has completed execution, its result is placed in the RF. This process is called instruction committment $(I C)$. The $I C$ stage basically signifies the termination of the instruction processing. Note that while instructions may complete execution out of order, they are committed in program order. For the purpose of this discussion and its related model, we can view the IQ and ROB as a single block where instructions are buffered in order according to their issue (arrival) order from the instruction fetch unit of the processor.

Memory instructions such as load and store instructions are directed to the Load/Store Queue (L/SQ) shown in Figure 15. Consider the execution of a load instruction. First an attempt is made to access the data from the data cache. If that is successful (cache hit), the data is read from the cache line and sent to the corresponding instruction entry in ROB. If the requested data is not in the cache (cache miss) it has to be fetched from the next level of the memory hierarchy, for example main memory, typically DRAM. In that case a 


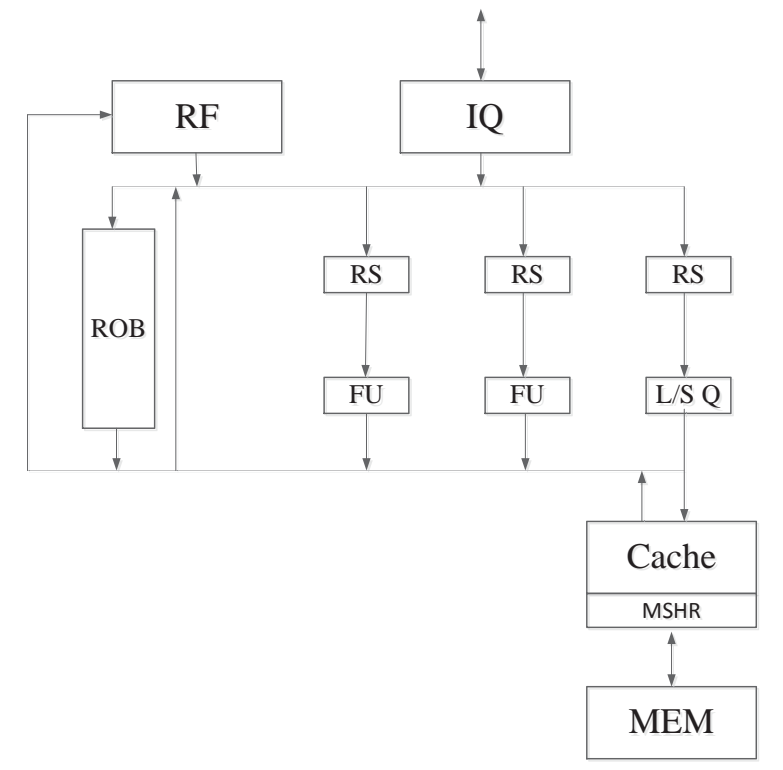

Figure 15: High-level OOO architecture

request is sent to the Miss Status Handling Register (MSHR), which serves as a finite-buffer queue for buffering outstanding non-cache memory access requests. When the MSHR is full all new instructions that generate memory-accesses are stalled. Upon completion of the memory access, the data is sent to the ROB and the instruction clears the MSHR. Note that the MSHR is a finite-buffer queue that holds only non-cache memory instructions. However, when it becomes full, it halts all new memory access requests, and thus comprises a fairly nonstandard queueing model.

To quantify all of this (see also [9]), consider a control cycle comprised of instructions $I_{i}, i=1, \ldots, M$ for a given $M>0$. Let us set the time to $t=0$ at the moment the first instruction is issued. In the framework of the closedloop system defined by the regulation algorithm, the control variable $u$ will be the clock frequency (rate) of the core, but it is easier to carry out the IPA computations in terms of the clock cycle, $\tau:=u^{-1}$.

For instruction $I_{i}$, define $\xi_{i}$ to be its issue time in terms of clock cycles (counting from the issue time of $\left.I_{1}\right) ; \alpha_{i}(\tau)$ - its execution starting time (in seconds), and $\beta_{i}(\tau)$ - its completion time (in seconds), not to be confused with its commit time which may occur later. Note that we use two kinds of timing variables, namely in units of clock cycles or seconds. The former can be measured in real time for the purpose of the control law, while the latter are used only in the analysis.

Consider first the case where $I_{i}$ is not a memory instruction; memory instructions will be handled later. The issue time of $I_{i}$ (in seconds) is $\xi_{i} \tau$, and 
we assume that there are available resources in the ROB and RS so that the instruction is forwarded there at the same time, $\xi_{i} \tau$. If $I_{i}$ is data dependent, denote by $k(i)$ the index (counter) of the instruction which is the last to provide a variable to $I_{i}$. Then, we have that

$$
\alpha_{i}(\tau)= \begin{cases}\max \left\{\xi_{i} \tau, \beta_{k(i)}(\tau)\right\}+\tau, & \text { if } I_{i} \text { is data dependent } \\ \xi_{i} \tau+\tau, & \text { otherwise. }\end{cases}
$$

As for the completion time, let $\mu_{i}$ denote the execution time of $I_{i}$ in terms of clock cycles. Then,

$$
\beta_{i}(\tau)=\alpha_{i}(\tau)+\mu_{i} \tau
$$

Consider next the case where $I_{i}$ is a memory instruction. Upon its issuance at time $\xi_{i} \tau$ it is directed to the L/SQ where a cache attempt is made. Let us regard the starting time of the cache attempt as the starting time of the instruction's execution at the memory stage, and denote it by $\alpha_{i}(\tau)$. If the MSHR is full at time $\xi_{i} \tau$, let $\ell(i)$ denote the index of the instruction at the head of the MSHR. Then,

$$
\alpha_{i}(\tau)= \begin{cases}\xi_{i} \tau+\tau, & \text { if MSHR is not full at time } \xi_{i} \tau \\ \beta_{\ell(i)}(\tau)+\tau, & \text { if MSHR is full at time } \xi_{i} \tau .\end{cases}
$$

Let $\nu_{i}$ denote the time (in units of clock cycles) it takes the L/SQ to process $I_{i}$ including the cache attempt. If the cache attempt result is successful (cache hit), then

$$
\beta_{i}(\tau)=\alpha_{i}(\tau)+\nu_{i} \tau
$$

On the other hand, in case of a cache miss, the instruction is directed to the MSHR for non-cache memory access. Let $m_{i}$ denote the processing time of the instruction in the MSHR in units of clock cycles, and let $M E M_{i}$ be the time it takes to access the memory. Note that DRAM access is not governed by the core's clock and hence it is not a function of $\tau$. Let $j(i)$ denote the index of the instruction prior to $I_{i}$ in the MSHR. Then, the completion time of $I_{i}$ is given by

$$
\beta_{i}(\tau)=\max \left\{\alpha_{i}(\tau)+\nu_{i} \tau+m_{i} \tau+M E M_{i}, \beta_{j(i)}(\tau)+\tau\right\} .
$$

Finally, the commit (departure) times of instructions $I_{i}, i=1,2, \ldots$, denoted by $d_{i}(\tau)$, are given by the following recursive equation,

$$
d_{i}(\tau)=\max \left\{\beta_{i}(\tau), d_{i-1}(\tau)\right\}+\tau .
$$

Now recall that the control cycle consists of $M$ instructions, and define the average throughput by

$$
y:=L(u)=\frac{M}{d_{M}(u)} .
$$

With $u=\tau^{-1}$, its IPA derivative is

$$
L^{\prime}(u)=\frac{1}{M}\left(\frac{y}{u}\right)^{2} d_{M}^{\prime}(\tau),
$$


where we assume that the throughput $y=L(u)$ can be computed from the system. The IPA term $d_{M}^{\prime}(\tau)$ is computable in a recursive manner as follows. By Equations (27)-(32), for every $i=1, \ldots, M$ : If $I_{i}$ is not a memory instruction, then

$$
\alpha_{i}^{\prime}(\tau)= \begin{cases}\beta_{k(i)}^{\prime}(\tau)+1, & \text { if } I_{i} \text { is stalled due to data dependency } \\ \xi_{i}+1, & \text { otherwise, }\end{cases}
$$

and

$$
\beta_{i}^{\prime}(\tau)=\alpha_{i}^{\prime}(\tau)+\mu_{i}
$$

On the other hand, if $I_{i}$ is a memory instruction, then

$$
\alpha_{i}^{\prime}(\tau)= \begin{cases}\beta_{\ell(i)}^{\prime}(\tau)+1, & \text { if } I_{i} \text { is stalled due to full MSHR } \\ \xi_{i}+1, & \text { otherwise }\end{cases}
$$

as for $\beta_{i}^{\prime}(\tau)$, if $I_{i}$ results in a cache hit, then

$$
\beta_{i}^{\prime}(\tau)=\alpha_{i}^{\prime}(\tau)+\nu_{i}
$$

and in the event of a cache miss,

$$
\beta_{i}^{\prime}(\tau)= \begin{cases}\alpha_{i}^{\prime}(\tau)+\nu_{i}+m_{i}, & \text { if } I_{i} \text { is first in the MSHR queue by the time } \\
\beta_{j(i)}(\tau)+1, & \begin{array}{l}
\text { its variable is read from memory } \\
\text { otherwise }
\end{array}\end{cases}
$$

It is reasonable to assume that the quantities $\xi_{i}, \mu_{i}, \nu_{i}$, and $m_{i}$ can be read from the system in real time during instruction execution, and hence the computation of $\beta_{i}^{\prime}(\tau), i=1, \ldots$, can be performed in real time in a recursive fashion via Equations (35)-(39). Finally, by (32),

$$
d_{i}^{\prime}(\tau)= \begin{cases}d_{i-1}^{\prime}(\tau)+1, & \text { if } I_{i} \text { is stalled in ROB following its execution } \\ \beta_{i}^{\prime}(\tau)+1, & \text { if } I_{i} \text { is comitted right after its execution. }\end{cases}
$$

This yields $d_{M}^{\prime}(\tau)$, and hence $L^{\prime}(u)$ via Equation (34). We point out that this IPA derivative is biased due to the fact that the DRAM and other non-cache memory accesses are not controlled by the core's clock - the memory system is in a different clock domain. We note that the time required for such memory access typically is one order of magnitude longer than a cache-access time and can be two orders of magnitude longer than computing instructions. Therefore we expect the regulation technique to perform better when applied to computationintensive programs rather than to memory-intensive programs. This is evident from the simulation results which will be presented in the following paragraphs.

We implemented the control algorithm using Manifold, a cycle-level, full system discrete event simulation platform for multi-core architectures [29]. A Manifold model boots a Linux operating system and executes stock 32-bit x86 binaries. Our experiments were applied to two programs from the SPLASH-2 suite of benchmark programs [27], Barnes and Radiosity. Barnes is compute 
intensive while Radiosity is memory intensive. For both cases the Manifold processor model used is similar to the Intel Nehalem micro-architecture comprised of four cores, each having its own L1 cache and sharing an L2 cache [23]. Each core is in a separate clock domain that is independently controlled. At the start of the simulation the application is emulated for a million instructions (out of an order of $10^{12}$ instructions) in order to warm up the architecture state. At this point, cycle-level timing simulation is begun over program regions of interest.

The control cycle for each core consists of $M=10,000$ instructions. Thus, for a given core, the control variable during the $n t h$ cycle is $u_{n}$, and $y_{n}$ is the instruction throughput computed at the end of the cycle via Eq. (33) (with the index $n$ added). The IPA derivative is computed via Eq. (34). It is not unbiased since the sample-performance function $L(u)$ is not necessarily continuous. This is largely due to the possibility of instruction stalls when the MSHR queue becomes full; see [11] for the relation between discontinuous sample performance functions and the biasedness of IPA. However, we believe that the error introduced by the bias generally is not large enough to prevent convergence of the regulation algorithm. Therefore, in contrast with the case of the finite-buffer queue discussed in Section 3.2, we do not resort to a fluid queueing model but rather compute the IPA derivatives directly from the discrete model according to Eqs. (34) - (40).

In the simulation experiments we took the target instruction rates (setpoints) for Cores 0-3 to be 1.0 Giga Instruction Per Second (GIPS), 1.5 GIPS, 2.0 $G I P S$, and 2.5 GIPS, respectively. Figure 16 shows simulation results for the benchmark Barnes executing at all four cores, and they indicate convergencetimes of the algorithm between $0.02 \mathrm{~ms}$ (Core 3) and $0.08 \mathrm{~ms}$ (Core 2). ${ }^{4}$ The apparent oscillations after convergence are due to variations in the programs' instruction loads, and their magnitudes are within $10 \%$ of the respective target values. However, the average instruction rates from time $0.1 \mathrm{~ms}$ to the final time $(0.25 \mathrm{~ms})$ are $0.9998,1.5025,1.9997$, and 2.4985 , which are within $0.2 \%$ of the respective target values.

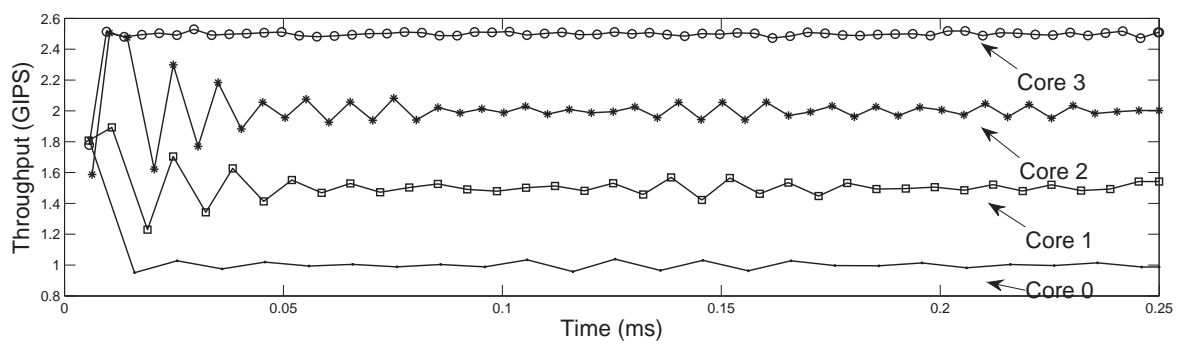

Figure 16: Instruction rate regulation: Barnes

\footnotetext{
${ }^{4}$ Using a cruder model, Reference [2] reports convergence in about $1 \mathrm{~ms}$.
} 
For the Radiosity benchmark we set the target instruction rates for Cores 0-3 to 1.0 GIPS, 1.3 GIPS, 1.5 GIPS, and 1.7 GIPS, respectively. Typical results are shown in Figure 17, where we discern convergence times between $0.12 \mathrm{~ms}$ to $0.14 \mathrm{~ms}$, with subsequent oscillations below $15 \%$ of the respective target values. The slower convergence as compared to Barnes is due to the fact that Radiosity is more memory intensive.

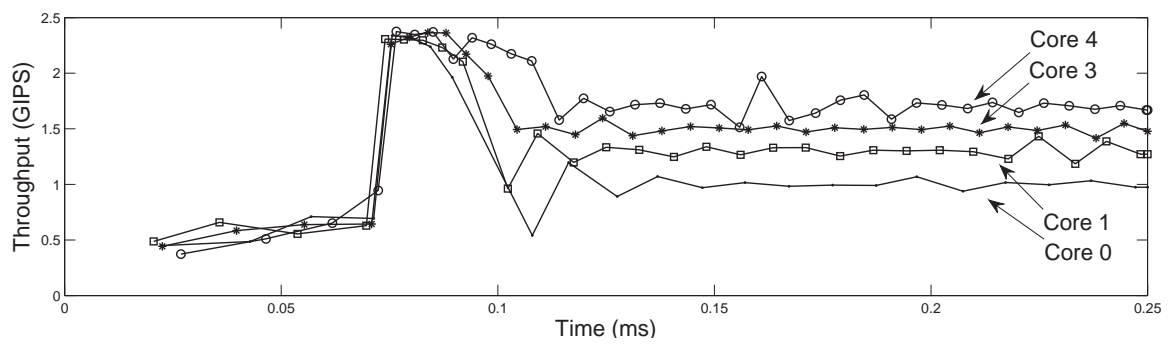

Figure 17: Instruction rate regulation: Radiosity

One way to reduce the oscillations is to scale the integrator's gain in Equation (1) by a constant $k \in(0,1)$, thereby replacing (1) by the following equation,

$$
u_{n}=u_{n-1}+k A_{n} e_{n-1} .
$$

After some experimentation we chose $k=0.2$. This resulted in reductions in the oscilations' magnitudes from $10 \%$ to $5 \%$ for Barnes, and from $15 \%$ to $10 \%$ for radiosity. The results are shown in Figures 18 and 19, respectively.

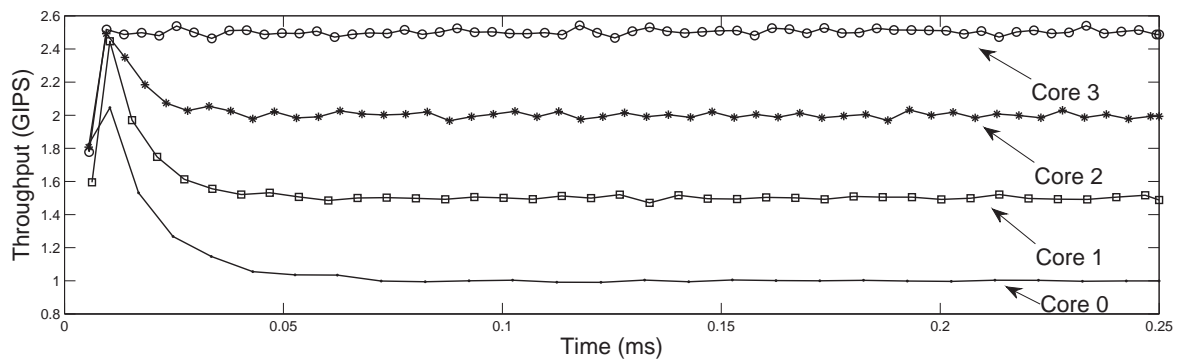

Figure 18: Instruction rate regulation (modified algorithm): Barnes

\section{Conclusions}

This paper describes an IPA-based approach to performance regulation in stochastic timed discrete event dynamic systems. The considered problem is to 


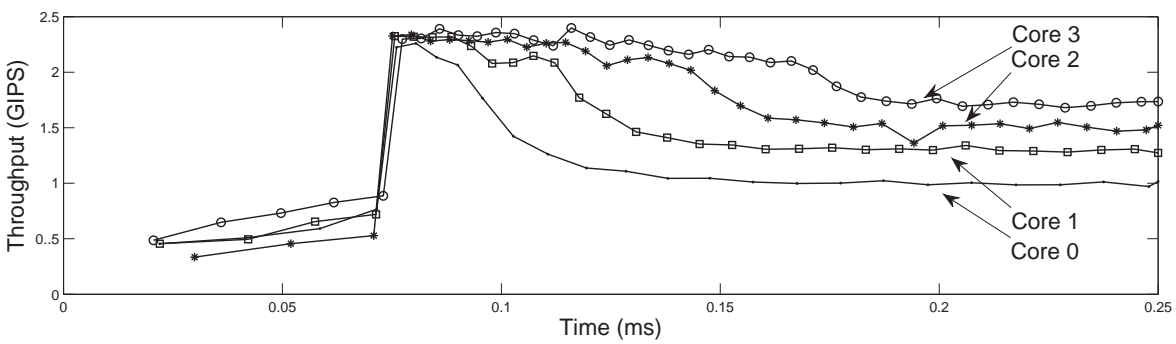

Figure 19: Instruction rate regulation (modified algorithm): Radiosity

control the output of the system so as to have it track (asymptotically) a given performance reference in the face of variations in the system's characteristics. The proposed approach is based on an integrator with adaptive gain. The system's plant is represented via a discrete-event or hybrid model, and the controller's gain is inverse proportional to the IPA derivative of its plant function with respect to the control parameter.

The paper summarizes the regulation technique and presents several examples, which highlight the fact that it can work well despite errors in estimating the IPA gradient. The examples include delay and loss in a single queue, inventory control in a Petri-net model of a production system, and throughput regulation in a computer core. Extensions and future investigations will focus on emerging problems in various application areas.

\section{Appendix}

This section provides proofs to Lemma 2.2 and Proposition 2.3 .

Proof of Lemma 2.2. Consider a closed, finite-length interval $I$ where $g(\cdot)$ is monotone-nondecreasing and convex throughout $I$. We next prove the assertion of the lemma for this case, while the situations where $g(\cdot)$ is monotone-nonincreasing or concave can be analyzed by similar arguments and hence their proofs are omitted.

By the monotonicity of $g(\cdot)$ we have that $g^{\prime}(u) \geq 0 \forall u \in I$, and we will use this fact throughout the forthcoming analysis. By convexity of $g(\cdot)$ we have the following inequalities for every $u \in I$ and $\Delta u \in R$ such that $u+\Delta u \in I$ :

$$
g(u)+g^{\prime}(u) \Delta u \leq g(u+\Delta u) \leq g(u)+g^{\prime}(u+\Delta u) \Delta u .
$$

Given $M \geq 1, \beta \in(0,1)$, and $\alpha \in(0,1)$, suppose that, for some $j=1, \ldots$, $u_{j-1} \in I, \mathcal{E}_{j-1}<\alpha$, and $\mathcal{G}_{j-1}<\beta$. We next derive upper bounds on the ratio $\mid g\left(u_{j}|/| g\left(u_{j-1}\right) \mid\right.$ (for the case where $g\left(u_{j-1}\right) \neq 0$ ) in terms of $M, \beta$, and $\alpha$. The analysis concerns four separate cases: (i) $g\left(u_{j-1}\right) \leq 0$ and $g\left(u_{j}\right) \leq 0$; (ii) $g\left(u_{j-1}\right) \leq 0$ and $g\left(u_{j}\right) \geq 0$; (iii) $g\left(u_{j-1}\right) \geq 0$ and $g\left(u_{j}\right) \leq 0$; and (iv) $g\left(u_{j-1}\right) \geq 0$ and $g\left(u_{j}\right) \geq 0$. The results, to be derived in the following paragraph, are: 
- Case (i):

$$
\left|g\left(u_{j}\right)\right| \leq\left(1-\frac{1-\beta}{1+\alpha}\right)\left|g\left(u_{j-1}\right)\right| .
$$

- Case (ii):

$$
\left|g\left(u_{j}\right)\right| \leq\left(M \frac{1+\beta}{1-\alpha}-1\right)\left|g\left(u_{j-1}\right)\right| .
$$

- Case (iii):

$$
\left|g\left(u_{j}\right)\right| \leq\left(\frac{1+\beta}{1-\alpha}-1\right)\left|g\left(u_{j-1}\right)\right| .
$$

- Case (iv):

$$
\left|g\left(u_{j}\right)\right| \leq\left(1-\frac{1}{M} \frac{1-\beta}{1+\alpha}\right)\left|g\left(u_{j-1}\right)\right| .
$$

We next prove these inequalities. In all cases we consider Eq. (42) with $u=u_{j-1}$ and

$$
\Delta u=-\frac{1}{g^{\prime}\left(u_{j-1}\right)+\phi_{j-1}}\left(g\left(u_{j-1}\right)+\psi_{j-1}\right) .
$$

By Eq. (10), $u+\Delta u=u_{j}$.

Case (i): $g\left(u_{j-1}\right) \leq 0$ and $g\left(u_{j}\right) \leq 0$. The left inequality of Eq. (42) means that

$$
g\left(u_{j-1}\right)-g^{\prime}\left(u_{j-1}\right) \frac{1}{g^{\prime}\left(u_{j-1}\right)+\phi_{j-1}}\left(g\left(u_{j-1}\right)+\psi_{j-1}\right) \leq g\left(u_{j}\right),
$$

and with a straightforward algebra,

$$
g\left(u_{j}\right) \geq g\left(u_{j-1}\right)-\frac{1}{1+\frac{\phi_{j-1}}{g^{\prime}\left(u_{j-1}\right)}}\left(1+\frac{\psi_{j-1}}{g\left(u_{j-1}\right)}\right) g\left(u_{j-1}\right) .
$$

By definition of $\mathcal{E}_{j-1}$ and $\mathcal{G}_{j-1},\left|\frac{\phi_{j-1}}{g^{\prime}\left(u_{j-1}\right)}\right| \leq \mathcal{E}_{j-1}$ and $\left|\frac{\psi_{j-1}}{g\left(u_{j-1}\right)}\right| \leq \mathcal{G}_{j-1}$; and by assumption, $\mathcal{E}_{j-1} \leq \alpha$ and $\mathcal{G}_{j-1} \leq \beta$; hence, and by (48) and the assumption that $g\left(u_{j-1}\right) \leq 0$, we have that

$$
g\left(u_{j}\right) \geq\left(1-\frac{1-\beta}{1+\alpha}\right) g\left(u_{j-1}\right) .
$$

By the assumption that $g\left(u_{j}\right) \leq 0$, Eq. (43) follows.

Case (ii): $g\left(u_{j-1}\right) \leq 0$ and $g\left(u_{j}\right) \geq 0$. The right inequality of (42) means that

$$
g\left(u_{j}\right) \leq g\left(u_{j-1}\right)-\frac{g^{\prime}\left(u_{j}\right)}{g^{\prime}\left(u_{j-1}\right)+\phi_{j-1}}\left(g\left(u_{j-1}\right)+\psi_{j-1}\right),
$$

and multiplying and dividing the RHS of $(50)$ by $g^{\prime}\left(u_{j-1}\right)$ and $g\left(u_{j-1}\right)$ we obtain that

$$
g\left(u_{j}\right) \leq g\left(u_{j-1}\right)-\frac{g^{\prime}\left(u_{j}\right)}{g^{\prime}\left(u_{j-1}\right)} \frac{1}{1+\frac{\phi_{j-1}}{g^{\prime}\left(u_{j-1}\right)}}\left(1+\frac{\psi_{j-1}}{g\left(u_{j-1}\right)}\right) g\left(u_{j-1}\right) .
$$


By definition of $\mathcal{E}_{j-1}$ and $\mathcal{G}_{j-1},\left|\frac{\phi_{j-1}}{g^{\prime}\left(u_{j-1}\right)}\right| \leq \mathcal{E}_{j-1}$ and $\left|\frac{\psi_{j-1}}{g\left(u_{j-1}\right)}\right| \leq \mathcal{G}_{j-1}$; and by assumption, $\mathcal{E}_{j-1} \leq \alpha, \mathcal{G}_{j-1} \leq \beta$, and $\frac{g^{\prime}\left(u_{j}\right)}{g^{\prime}\left(u_{j-1}\right)} \leq M$; hence, and by (51) and the assumption that $g\left(u_{j-1}\right) \leq 0$, we have that

$$
g\left(u_{j}\right) \leq\left(1-M \frac{1+\beta}{1-\alpha}\right) g\left(u_{j-1}\right) .
$$

Since by assumption $g\left(u_{j-1}\right) \leq 0$ and $g\left(u_{j}\right) \geq 0$, Eq. (44) follows.

Case (iii): $g\left(u_{j-1}\right) \geq 0$ and $g\left(u_{j}\right) \leq 0$. As in Case (i), Eq. (48) is satisfied, and since $g\left(u_{j-1}\right) \geq 0$,

$$
g\left(u_{j}\right) \geq g\left(u_{j-1}\right)-\frac{1+\beta}{1-\alpha} g\left(u_{j-1}\right)=\left(1-\frac{1+\beta}{1-\alpha}\right) g\left(u_{j-1}\right) .
$$

Since $g\left(u_{j}\right) \leq 0$, Eq. (45) follows.

Case (iv): $g\left(u_{j-1}\right) \geq 0$ and $g\left(u_{j}\right) \geq 0$. As in the analysis for Case (ii), Eq. (51) applies, and by the definition of $M, \frac{g^{\prime}\left(u_{j}\right)}{g^{\prime}\left(u_{j-1}\right)} \geq \frac{1}{M}$. Therefore

$$
g\left(u_{j}\right) \leq\left(1-\frac{1}{M} \frac{1-\beta}{1+\alpha}\right) g\left(u_{j-1}\right)
$$

which is Eq. (46).

Fix $M>1$ and $\beta \in\left(0, M^{-1}\right)$. Consider $\alpha \in(0,1)$, and $n=1, \ldots$, and suppose that all of the conditions specified in the assertion of the lemma are satisfied. We next prove that Eq. (11) is satisfied for an $\alpha>0$ and a corresponding $\theta \in(0,1)$. There are two scenarios to consider: (a) $m_{n}=n+1$, and (b) $m_{n}>n+1$.

Scenario (a) means that $g\left(u_{n}\right) g\left(u_{n-1}\right) \geq 0$ and arises when either Case (i) or Case (iv) occur. In Case (i), Eq. (43) is in force, and since $\left.\left(1-\frac{1-\beta}{1+\alpha}\right)\right|_{\alpha=0}=$ $\beta<1$, it follows that there exists $\alpha_{1} \in(0,1)$ and $\theta_{1} \in(0,1)$ such that, for every $\alpha \in\left(0, \alpha_{1}\right)$, Eq. (11) is satisfied with $\theta_{1}$ in lieu of $\theta$. Similarly in Case (iv) and Eq. (46); since $\left.\left(1-\frac{1}{M} \frac{1-\beta}{1+\alpha}\right)\right|_{\alpha=0}=1-\frac{\beta}{M}<1$, there exists $\alpha_{4} \in(0,1)$ and $\theta_{4} \in(0,1)$ such that, for every $\alpha \in\left(0, \alpha_{4}\right)$, Eq. (11) is satisfied with $\theta_{4}$ in lieu of $\theta$.

Scenario (b) corresponds to either Case (ii) or Case (iii). In Case (iii), where $g\left(u_{n-1}\right) \geq 0$ while $g\left(u_{n}\right) \leq 0$, Eq. (45) is satisfied. Note that $\left.\left(\frac{1+\beta}{1-\alpha}-1\right)\right|_{\alpha=0}=$ $\beta<1$, and therefore, there exists $\alpha_{3} \in(0,1)$ and $\theta_{3} \in(0,1)$ such that, if $\alpha \in\left(0, \alpha_{3}\right)$, then

$$
\left|g\left(u_{n}\right)\right| \leq \theta_{3}\left|g\left(u_{n-1}\right)\right| .
$$

In Case (ii), where $g\left(u_{n-1}\right) \leq 0$ while $g\left(u_{n}\right) \geq 0$, Eq. (44) holds, but unlike the other three cases, it is not true that $\left.\left(M \frac{1+\beta}{1-\alpha}-1\right)\right|_{\alpha=0}<1$. A different argument is needed.

Suppose first that Case (ii) holds at $u_{n-1}$, Case (iii) will be considered later. By definition of $m_{n}$, Case (iii) holds at $m_{n}-1$ while Case (iv) is satisfied for all $j=n, \ldots, m_{n}-2$. By Equations (44)-(46),

$$
\left|g\left(u_{n_{n-1}}\right)\right| \leq\left(M \frac{1+\beta}{1-\alpha}-1\right)\left(\frac{1+\beta}{1-\alpha}-1\right)\left(1-\frac{1}{M} \frac{1-\beta}{1+\alpha}\right)^{m_{n-1}-(n-1}\left|g\left(u_{n-1}\right)\right| .
$$


The above analysis of Case (iv) showed that if $\alpha<\alpha_{4}$ then $\left(1-\frac{1}{M} \frac{1-\beta}{1+\alpha}\right)<1$, and therefore,

$$
\left|g\left(u_{n_{n-1}}\right)\right| \leq\left(M \frac{1+\beta}{1-\alpha}-1\right)\left(\frac{1+\beta}{1-\alpha}-1\right)\left|g\left(u_{n-1}\right)\right|
$$

$\left.\operatorname{But}\left(M \frac{1+\beta}{1-\alpha}-1\right)\left(\frac{1+\beta}{1-\alpha}-1\right)\right|_{\alpha=0}=(M(1+\beta)-1) \beta<M \beta<1$, where the last two inequalities are due to the assumption that $M \beta<1$. Therefore, there exist $\alpha_{2} \in(0,1)$ and $\theta_{2} \in(0,1)$ such that, if $\alpha<\alpha_{2}$, Eq. (11) is satisfied with $\theta_{2}$ in lieu of $\theta$.

Finally, in Case (iii) at $u_{n-1}$, Eq. (57) is provable in the same way as for Case (ii), and the conclusion is derivable in the same way as well.

Now by defining $\alpha=\min \left\{\alpha_{i}: i=1, \ldots, 4\right\}$, Eq. (11) is satisfied with $\theta:=\max \left\{\theta_{i}: i=1, \ldots, 4\right\}$. This completes the proof.

Proof of Proposition 2.3. Given $\eta>0, M>1$, and $\varepsilon>0$. Fix $\beta \in\left(0, M^{-1}\right)$. Choose $\alpha \in(0,1)$ and $\theta \in(0,1)$ according to Lemma 2.2. In particular, as in the proof of Lemma 2.2 we can assume, by reducing $\alpha$ is necessary, that

$$
\left(M \frac{1+\beta}{1-\alpha}-1\right)\left(\frac{1+\beta}{1-\alpha}-1\right)<1 .
$$

Consider a closed, finite-length interval $I$ and a sequence of points $\left\{u_{n}\right\}_{n=1}^{\infty}$ satisfying the conditions of the proposition. Suppose, without loss of generality, that $g(\cdot)$ is monotone nondecreasing and convex on $I$. Since $\left|g^{\prime}(u)\right| \geq \eta$ for every $u \in I$, it follows by Eq. (10) that there exists $K>0$ (independent of $I$ or the sequence $\left.\left\{u_{n}\right\}\right)$ such that, for every $n=1, \ldots$,

$$
\left|g\left(u_{n}\right)\right| \leq K\left|g\left(u_{n-1}\right)\right|
$$

Fix $\varepsilon^{\prime}>0$ such that

$$
2 K \varepsilon^{\prime}<\varepsilon
$$

Fix $\delta>0$ such that

$$
\delta<\min \left\{\beta \varepsilon^{\prime}, \frac{\varepsilon}{2}\right\} .
$$

By assumption $\mathcal{E}_{n}<\alpha$ and $\left|\psi_{n}\right|<\delta$ for all $n=1, \ldots$, . As a result of the inequality $\left|\psi_{n}\right|<\delta$, and by Eq. (61), if $\mathcal{G}_{n}:=\frac{\left|\psi_{n}\right|}{\left|g\left(u_{n}\right)\right|}>\beta$ then $\left|g\left(u_{n}\right)\right|<\varepsilon^{\prime}$. Thus, if $\left|g\left(u_{n}\right)\right|>\varepsilon^{\prime}$ then Eqs. (43)-(46) hold with $n+1$ in lieu of $j$, and if $\left|g\left(u_{n}\right)\right|<\varepsilon^{\prime}$ then

$$
\left|g\left(u_{n+1}\right)\right| \leq K \varepsilon^{\prime}
$$

Now consider a point $u_{n}$ such that $\left|g\left(u_{n}\right)\right|>\varepsilon^{\prime}$ and hence $\mathcal{G}_{n} \leq \beta$. Consider the four cases (i) - (iv) in the proof of Lemma 2.2. In Case (i) and Case (iv), $m_{n}=n+1$, and Eq. (11) implies that $\left|g\left(u_{n+1}\right)\right|<\left|g\left(u_{n}\right)\right|$. In Case (iii), Eq. (55) implies the same inequality, namely $\left|g\left(u_{n+1}\right)\right|<\left|g\left(u_{n}\right)\right|$. Only in Case (ii) the reverse inequality is possible, namely that $\left|g\left(u_{n+1}\right)\right| \geq\left|g\left(u_{n}\right)\right|$. 
Suppose that Case (ii) holds at $u_{n}$. Then (by definition of Case (ii)) $g\left(u_{n}\right) \leq$ $0, g\left(u_{j}\right) \geq 0 \forall j=n+1, \ldots, m_{n}-1$, and $g\left(u_{m_{n}}\right) \leq 0$. Moreover, for every $j=$ $n+1, \ldots, m_{n}-1$, either Case (iii) or (iv) holds, and therefore, either $\left|g\left(u_{j+1}\right)\right|<$ $\left|g\left(u_{j}\right)\right|$ if $\left|g\left(u_{j}\right)\right| \geq \varepsilon^{\prime}$, or $\left|g\left(u_{j+1}\right)\right| \leq K \varepsilon^{\prime}$ if $\mid\left(g\left(u_{j}\right) \mid \leq \varepsilon^{\prime}\right.$. Consequently, and by Eq. (62), we have that

$$
\left|g\left(u_{m_{n}}\right)\right| \leq \max \left\{K \varepsilon^{\prime},\left|g\left(u_{n+1}\right)\right|\right\} .
$$

As a result we have the following situation: If $\left|g\left(u_{j}\right)\right| \leq \varepsilon^{\prime}$ for some $j=$ $n, \ldots, m_{n}-1$, then $\left|g\left(u_{m_{n}}\right)\right| \leq K \varepsilon^{\prime}$. On the other hand, if $\left|g\left(u_{j}\right)\right| \geq \varepsilon^{\prime}$ for every $j=n, \ldots, m_{n}-1$, then (by Lemma 2.2), Eq. (11) is in force. This, in conjunction with Eq. (61), implies that

$$
\limsup _{n \rightarrow \infty}\left|g\left(u_{n}\right)\right| \leq K \varepsilon^{\prime} \leq \frac{\varepsilon}{2}
$$

hence the inequality in Eq. (13).

Finally, Eq. (14) follows from (13) and the assumption that $\delta<\frac{\varepsilon}{2}$, as specified in Eq. (61). This completes the proof.

\section{References}

[1] Almoosa, N., Song, W., Wardi, Y., and Yalamanchili, S. (2012). A Power Capping Controller for Multicore Processors. Proc. 2012 American Control Conference, Montreal, Canada, June 27-29.

[2] Almoosa, N., Song, W., Wardi, Y., and Yalamanchili, S. (2012a). Throughput Regulation in Multicore Processors via IPA. Proc. 51 IEEE Conference on Decision and Control (CDC), Maui, Hawaii, December 10-13.

[3] Bauer, M., Pacher, M., and Brinkschulte, U. (2010). A Chip-size Evaluation of a Multi-threaded Processor Enhanced with a PID Controller. Proc. 8th IFIP Workshop on Software Technologies for Future Embedded and Ubiquitous Systems (SEUS 2010), Waidhofen, Austria, October 13-15, 2010.

[4] Brinkschulte, U., and Pacher, M. A Theoretical Examination of a SelfAdaptation Approach to Improve the Real-Time Capabilities in MultiThreaded Microprocessors. Proc. 2009 Third IEEE International Conference on Self-Adaptive and Self-Organizing Systems, San Francisco, California, September 14-18, 2009.

[5] Cassandras, C., and Lafortune, S. (1999). Introduction to Discrete Event Systems. Kluwer Academic Publishers, Boston, Massachusetts.

[6] Cassandras, C.G., Wardi, Y., Melamed, B., Sun, G., and Panayiotou, C.G. (2002). Perturbation Analysis for On-Line Control and Optimization of Stochastic Fluid Models. IEEE Transactions on Automatic Control, Vol. 47, No. 8, pp. 1234-1248. 
[7] Cassandras, C.G. (2006). Stochastic flow systems: Modeling and sensitivity analysis. In Stochastic Hybrid Systems: Recent Developments and Research Trends, Eds. C.G. Cassandras and J. Lygeros, CRC Press, New York, New York, pp. 137-165.

[8] Cassandras, C.G., Wardi, Y., Panayiotou, C.G., and Yao, C. (2010). Perturbation Analysis and Optimization of Stochastic Hybrid Systems. European Journal of Control, Vol. 16, pp. 642-664.

[9] Chen, X., Xiao, H., Wardi, Y., and Yalamanchili, S. (2015). Throughput Regulation in Shared Memory Multicore Prtocessors. Proc. 22nd IEEE Intl. Conference on High Performance Computing (HiPC), Bengaluru, India, December 16-19.

[10] Hennessey, J.L., and Patterson, D.A. (2012). Computer Architecture: A Quantitative Approach, Morgan Kaufmann.

[11] Ho, Y.C., and Cao, X.R. (1991). Perturbation Analysis of Discrete Event Dynamic Systems. Kluwer Academic Publishers, Boston, Massachusetts.

[12] Howard, J., Dighe, S., Vangal, S.R., Ruhl, G., Borkar, N., Jain, S., Erraguntla, V., Konow, M. Ripen, M. Gries, M., Droege, G., Lund-Larsen, T., Steibl, S. Borkar, S., De, V.K., and Van Det Wijngaart, R. A. (2011). 48-Core IA-32 Processor in $45 \mathrm{~nm}$ CMOS Using On-Die Message Passing and DVFS for Performance and Power Scaling. J. Solid State Circuits, Vol. 46, pp. 173-183.

[13] Kim, W., Gupta, M.S., Wei, G.-Y., and Brooks, D. (2008). System Level Analysis of Fast, Per-Core DVFS using On-Chip Switching Regulators. Proc. IEEE Intl. Symposium on High Performance of Computer Architectures.

[14] Lancaster, P. Error analysis for the Newton-Raphson method. Numerische Mathematik, Vol. 9, pp. 55-68, 1966.

[15] Lohn, D., Pacher, M., and Brinkschulte, U. A Generalized Model to Control the Throughput in a Processor for Real-Time Applications. 2011 14th IEEE International Symposium on Object/Component/Service-Oriented Real-Time Distributed Computing, Newport Beach, California, March 2831, 2011.

[16] Ortega, J.M., and Rheinboldt, W.C. (1970). Iterative Solutions of Nonlinear Equations in Several Variables. Academic Press, San Diego, California.

[17] Panayiotou, C.G., and Cassandras, C.G. (2006). Infinitesimal Perturbation Analysis and Optimization for Make-To-Stock Manufacturing Systems Based on Stochastic Fluid Models. Discrete Event Dynamic Systems: Theory and Applications, Vol. 16, pp. 109-142. 
[18] Seatzu, C., and Wardi, Y. (2013). On the Use of IPA in Performance Optimization of Continuous Marked Graphs: A Case Study. Proc. IEEE Conf. on Emerging Technologies and Factory Automation (ETFA'13), Cagliari, Italy, September 10-13.

[19] Seatzu, C., and Wardi, Y. (2014). Performance Regulation via Integral Control in a Class of Stochastic Discrete Event Dynamic Systems. Proc. 12th IFAC - IEEE International Workshop on Discrete Event Systems (WODES'14), Paris, France, May 14-16.

[20] Shakkottaia, S., and Stolyarb, A.L. (2001). Scheduling algorithms for a mixture of real-time and non-real-time data in HDR. Teletraffic Science and Engineering, Vol. 4, pp. 798-804.

[21] Silva, M. and Recalde, L. (2004). On fluidification of Petri net models: from discrete to hybrid and continuous models. Annual Reviews in Control, Vol. 28, pp. 253-266.

[22] Sun, G., Cassandras, C.G., Wardi, Y., Panayiotou, C.G., and Riley, G. (2004). Perturbation Analysis and Optimization of Stochastic Flow Networks. IEEE Transactions on Automatic Control, Vol. 49, No. 12, pp. 2143-2159.

[23] Thomadakis, M.E. (2011). The Architecture of the Nehalem Processor and Nehalem-EP SMP Platforms. E-PRINT Network (pubs.), pp. 2-5.

[24] Wardi, Y., Adams, R., and Melamed, B. (2010). A Unified Approach to Infinitesimal Perturbation Analysis in Stochastic Flow Models: The SingleStage Case. IEEE Transactions on Automatic Control, Vol. AC-55, No. 1, pp. 89-103.

[25] Wardi, Y., and Cassandras, C.G. (2014). Perturbation Analysis of Discrete Event Systems. in Encyclopedia on Systems and Control, Eds. T. Samad and J. Baillieul, Section on Discrete Event Systems, Ed. C.G. Cassandras.

[26] Wardi, Y., Giua, A., and Seatzu, C.(2013b). IPA for Continuous Stochastic Marked Graphs. Automatica, Vol. 49, No. 5, pp. 1204-1215.

[27] Woo, S.C., Oharat, M., Torriet, E., et al. (1995). The SPLASH-2 Programs: Characterization and Methodological Considerations. ISCA '95 Proceedings of the 22nd annual international symposium on Computer architectures, pp. 24-36.

[28] Xie, X. (2002). Fluid Stochastic Event Graphs for Evoluation and Optimization of Discrete-Event Systems with Failurs. IEEE Transactions on Robotics and Automation, Vol. 18, pp. 360-367.

[29] Yalamanchili, S., Riley, G., and Conte, T.M. http://manifold.gatech.edu/. 